\title{
REFINEMENTS OF YOUNG'S INTEGRAL INEQUALITY VIA FUNDAMENTAL INEQUALITIES AND MEAN VALUE THEOREMS FOR DERIVATIVES
}

\author{
FENG QI, WEN-HUI LI, GUO-SHENG WU, AND BAI-NI GUO \\ Dedicated to people facing and fighting 2019-nCoV
}

\begin{abstract}
In the paper, the authors review several refinements of Young's integral inequality via several mean value theorems, such as Lagrange's and Taylor's mean value theorems of Lagrange's and Cauchy's type remainders, and via several fundamental inequalities, such as Čebyšev's integral inequality, Hermite-Hadamard's type integral inequalities, Hölder's integral inequality, and Jensen's discrete and integral inequalities, in terms of higher order derivatives and their norms, survey several applications of several refinements of Young's integral inequality, and further refine Young's integral inequality via Pólya's type integral inequalities.
\end{abstract}

\section{Contents}

1. Young's integral inequality and several refinements 2

1.1. Young's integral inequality

1.2. Refinements of Young's integral inequality via Lagrange's mean value theorem

1.3. Refinements of Young's integral inequality via Hermite-Hadamard's and Čebyšev's integral inequalities

1.4. Refinements of Young's integral inequality via Jensen's discrete and integral inequalities

1.5. Refinements of Young's integral inequality via Hölder's integral inequality

1.6. Refinements of Young's integral inequality via Taylor's mean value theorem of Lagrange's type remainder

1.7. Refinements of Young's integral inequality via Taylor's mean value theorem of Cauchy's type remainder and Hölder's integral inequality

1.8. Refinements of Young's integral inequality via Taylor's mean value theorem of Cauchy's type remainder and Cebyšev's integral inequality

2010 Mathematics Subject Classification. Primary 26D15; Secondary 26A42, 26A48, 26A51, 26D05, 26D07, 33B10, 33B20, 41A58.

Key words and phrases. refinement; Young's integral inequality; monotonic function; inverse function; convex function; Pólya's type integral inequality; Lagrange's mean value theorem; Taylor's mean value theorem; Lagrange's type remainder; Cauchy's type remainder; higher order derivative; Čebyšev's integral inequality; Hermite-Hadmard's integral inequality; Hölder's integral inequality; Jensen's inequality; norm. 
1.9. Refinements of Young's integral inequality via Taylor's mean value theorem of Cauchy's type remainder and Jensen's inequalities 17

1.10. Refinements of Young's integral inequality via Taylor's mean value theorem of Cauchy's type remainder and integral inequalities of Hermite-Hadamard type for the product of two convex functions

1.11. Three examples showing refinements of Young's integral inequality [19]

2. New refinements of Young's integral inequality via Pólya's type integral inequalities

2.1. Refinements of Young's integral inequality in terms of bounds of the first derivative

2.2. Refinements of Young's integral inequality in terms of bounds of the second derivative

2.3. Refinements of Young's integral inequality in terms of bounds of

higher order derivatives
2.4. Refinements of Young's integral inequality in terms of $L^{p}$-norms

2.5. Three examples for new refinements of Young's integral inequalities

3. More remarks

References

\section{YOUNG'S INTEGRAL INEQUALITY AND SEVERAL REFINEMENTS}

In the first part of this paper, we mainly review several refinements of Young's integral inequality via several mean value theorems, such as Lagrange's and Taylor's mean value theorems of Lagrange's and Cauchy's type remainders, and via several fundamental inequalities, such as Čebyšev's integral inequality, HermiteHadamard's type integral inequalities, Hölder's integral inequality, and Jensen's discrete and integral inequalities, in terms of higher order derivatives and their norms, and simply survey several applications of several refinements of Young's integral inequality.

1.1. Young's integral inequality. One of fundamental and general inequalities in mathematics is Young's integral inequality below.

Theorem $1.1([54])$. Let $h(x)$ be a real-valued, continuous, and strictly increasing function on $[0, c]$ with $c>0$. If $h(0)=0, a \in[0, c]$, and $b \in[0, h(c)]$, then

$$
\int_{0}^{a} h(x) \mathrm{d} x+\int_{0}^{b} h^{-1}(x) \mathrm{d} x \geq a b,
$$

where $h^{-1}$ denotes the inverse function of $h$. The equality in (1.1) is valid if and only if $b=h(a)$.

Proof. This proof is adapted from the proof of [25, Section 2.7, Theorem 1].

Set

$$
f(a)=a b-\int_{0}^{a} h(x) \mathrm{d} x
$$


and consider $b>0$ as a parameter. Since $f^{\prime}(a)=b-h(a)$ and $h$ is strictly increasing, one obtains

$$
f^{\prime}(a) \begin{cases}>0, & 0<a<h^{-1}(b) ; \\ =0, & a=h^{-1}(b) ; \\ <0, & a>h^{-1}(b) .\end{cases}
$$

This means that $f(a)$ has a maximum of $f$ at $a=h^{-1}(b)$. Therefore, it follows that

$$
f(a) \leq \max \{f(x)\}=f\left(h^{-1}(b)\right) .
$$

Integrating by parts gives

$$
f\left(h^{-1}(b)\right)=b h^{-1}(b)-\int_{0}^{h^{-1}(b)} h(x) \mathrm{d} x=\int_{0}^{h^{-1}(b)} x h^{\prime}(x) \mathrm{d} x .
$$

Substituting $y=h(x)$ into the above integral yields

$$
f\left(h^{-1}(b)\right)=\int_{0}^{b} h^{-1}(y) \mathrm{d} y .
$$

Putting 1.2 and $(1.4)$ into 1.3 results in 1.1 . The proof of Theorem 1.1 is complete.

Remark 1.1. The geometric interpretation of Young's integral inequality (1.1) can be demonstrated by Figures 1 and 2

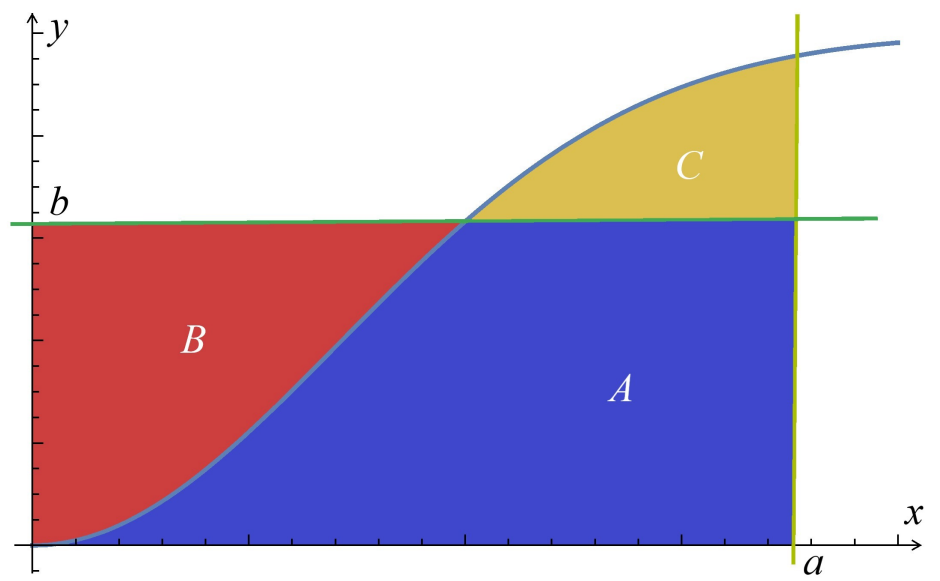

FIGURE 1. Geometric interpretation of the inequality (1.1)

In Figure 1, we have

$$
\begin{aligned}
& A+C=\int_{0}^{a} h(x) \mathrm{d} x, \quad A+B=a b, \quad B=\int_{0}^{b} h^{-1}(x) \mathrm{d} x, \\
& A+B+C=\int_{0}^{a} h(x) \mathrm{d} x+\int_{0}^{b} h^{-1}(x) \mathrm{d} x \geq a b=A+B .
\end{aligned}
$$

Therefore, the inequality 1.1 means that the area

$$
C=\int_{h^{-1}(b)}^{a} h(x) \mathrm{d} x-b\left[a-h^{-1}(b)\right] \geq 0 .
$$




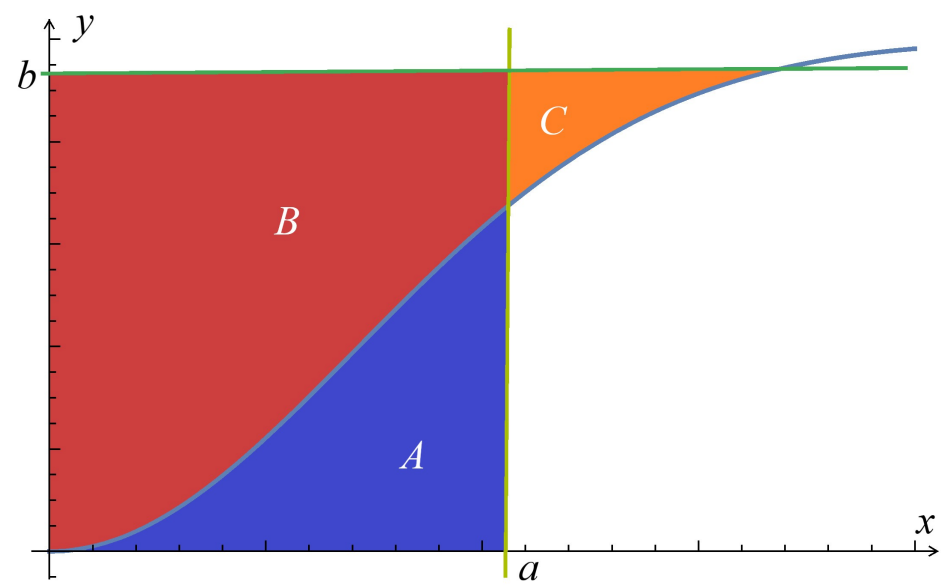

Figure 2. Geometric interpretation of the inequality (1.1)

In Figure 2, we have

$$
\begin{aligned}
& A=\int_{0}^{a} h(x) \mathrm{d} x, \quad A+B=a b, \quad B+C=\int_{0}^{b} h^{-1}(x) \mathrm{d} x, \\
& A+B+C=\int_{0}^{a} h(x) \mathrm{d} x+\int_{0}^{b} h^{-1}(x) \mathrm{d} x \geq a b=A+B .
\end{aligned}
$$

Therefore, the inequality 1.1 means that the area

$$
C=b\left[h^{-1}(b)-a\right]-\int_{a}^{h^{-1}(b)} h(x) \mathrm{d} x \geq 0 .
$$

Remark 1.2. We notice that two expressions $(1.5)$ and $(1.6)$ are of the same form

$$
C=\int_{h^{-1}(b)}^{a} h(x) \mathrm{d} x-a b+b h^{-1}(b) \geq 0,
$$

no matter which of $a$ and $h^{-1}(b)$ is smaller or bigger.

Remark 1.3. When $p>1$, taking $h(x)=x^{p-1}$ in 1.1 derives

$$
\frac{1}{p} a^{p}+\frac{1}{q} b^{q} \geq a b
$$

for $a, b \geq 0$ and $p, q>1$ satisfying $\frac{1}{p}+\frac{1}{q}=1$. Further replacing $a^{p}$ and $b^{q}$ by $x$ and $y$ respectively leads to

$$
x^{1 / p} y^{1 / q} \leq \frac{x}{p}+\frac{y}{q}
$$

for $x, y \geq 0$ and $a, q>1$ satisfying $\frac{1}{p}+\frac{1}{q}=1$. Perhaps this is why the weighted arithmetic-geometric inequality 1.8 is also called Young's inequality in [13, 14, 21] and closely related references therein.

Remark 1.4. The inequality

$$
\sum_{k=1}^{n} \frac{\cos (k \theta)}{k}>-1, \quad n \geq 2, \quad \theta \in[0, \pi]
$$


is also called Young's inequality in [2, 3] and closely related references therein.

Remark 1.5. In [25, Secton 2.7] and [26, Chapter XIV], a plenty of refinements, extensions, generalizations, and applications of Young's integral inequality (1.1) were collected, reviewed, and surveyed. For some new and recent development on this topic after 1990, please refer to the papers [4, 42, 46, 50, 55] and closely related references therein.

1.2. Refinements of Young's integral inequality via Lagrange's mean value theorem. In 2008, Hoorfar and Qi refined Young's integral inequality (1.1) via Lagrange's mean value theorem for derivatives.

Theorem $1.2([18$, Theorem 1]). Let $h(x)$ be a differentiable and strictly increasing function on $[0, c]$ for $c>0$ and let $h^{-1}$ be the inverse function of $h$. If $h(0)=0$, $a \in[0, c], b \in[0, h(c)]$, and $h^{\prime}(x)$ is strictly monotonic on $[0, c]$, then

$$
\frac{m}{2}\left[a-h^{-1}(b)\right]^{2} \leq \int_{0}^{a} h(x) \mathrm{d} x+\int_{0}^{b} h^{-1}(x) \mathrm{d} x-a b \leq \frac{M}{2}\left[a-h^{-1}(b)\right]^{2},
$$

where

$$
m=\min \left\{h^{\prime}(a), h^{\prime}\left(h^{-1}(b)\right)\right\}
$$

and

$$
M=\max \left\{h^{\prime}(a), h^{\prime}\left(h^{-1}(b)\right)\right\} .
$$

The equalities in 1.9 are valid if and only if $b=h(a)$.

Proof. This is a modification of the proof of [18, Theorem 1] in [18, Section 2].

Changing the variable of integration by $x=h(y)$ and integrating by parts yield

$$
\begin{aligned}
\int_{0}^{a} h(x) \mathrm{d} x+\int_{0}^{b} h^{-1}(x) \mathrm{d} x & =\int_{0}^{a} h(x) \mathrm{d} x+\int_{0}^{h^{-1}(b)} y h^{\prime}(y) \mathrm{d} y \\
& =\int_{0}^{a} h(x) \mathrm{d} x+b h^{-1}(b)-\int_{0}^{h^{-1}(b)} h(x) \mathrm{d} x \\
& =b h^{-1}(b)+\int_{h^{-1}(b)}^{a} h(x) \mathrm{d} x \\
& =a b+\int_{h^{-1}(b)}^{a}[h(x)-b] \mathrm{d} x .
\end{aligned}
$$

From the last line in 1.10 , we can see that, if $h^{-1}(b)=a$, then those equalities in $(1.9)$ hold.

If $h^{-1}(b)<a$, since $h(x)$ is strictly increasing, then $h(x)-b>0$ for $x \in$ $\left(h^{-1}(b), a\right)$. By Lagrange's mean value theorem for derivatives, we can see that there exists $\xi=\xi(x)$, satisfying $h^{-1}(b)<\xi<x \leq a$, such that

$$
0<h(x)-b=\left[x-h^{-1}(b)\right] h^{\prime}(\xi) .
$$

By virtue of monotonicity of $h^{\prime}(x)$ on $[0, c]$, we reveal that

$$
0<m=\min \left\{h^{\prime}(a), h^{\prime}\left(h^{-1}(b)\right)\right\}<h^{\prime}(\xi)<\max \left\{h^{\prime}(a), h^{\prime}\left(h^{-1}(b)\right)\right\}=M .
$$

Consequently, we have

$$
0<m\left[x-h^{-1}(b)\right]<h(x)-b<M\left[x-h^{-1}(b)\right] .
$$


As a result, we have

$$
m \int_{h^{-1}(b)}^{a}\left[x-h^{-1}(b)\right] \mathrm{d} x<\int_{h^{-1}(b)}^{a}[h(x)-b] \mathrm{d} x<M \int_{h^{-1}(b)}^{a}\left[x-h^{-1}(b)\right] \mathrm{d} x
$$

which is equivalent to

$$
\frac{m}{2}\left[a-h^{-1}(b)\right]^{2}<\int_{h^{-1}(b)}^{a}[h(x)-b] \mathrm{d} x<\frac{M}{2}\left[a-h^{-1}(b)\right]^{2} .
$$

If $h^{-1}(b)>a$, we can derive inequalities in (1.11) by a similar argument as above.

Substituting the double inequality 1.11 into the equality 1.10 leads to the double inequality 1.9 . The proof of Theorem 1.2 is complete.

Remark 1.6. The geometric interpretation of the double inequality 1.9 is that the areas $C$ in Figures 3 to 6 satisfy

$$
\frac{m}{2}\left[a-h^{-1}(b)\right]^{2} \leq C \leq \frac{M}{2}\left[a-h^{-1}(b)\right]^{2} .
$$

When $h^{\prime}(x)$ is strictly increasing, the double inequality 1.12 can be equivalently written as

$$
\frac{h^{\prime}(a)}{2}\left[a-h^{-1}(b)\right] \leq \frac{\int_{h^{-1}(b)}^{a} h(x) \mathrm{d} x}{a-h^{-1}(b)} \leq \frac{h^{\prime}\left(h^{-1}(b)\right)}{2}\left[a-h^{-1}(b)\right]
$$

and

$$
\frac{h^{\prime}(a)}{2}\left[h^{-1}(b)-a\right] \leq \frac{\int_{a}^{h^{-1}(b)} h(x) \mathrm{d} x}{h^{-1}(b)-a} \leq \frac{h^{\prime}\left(h^{-1}(b)\right)}{2}\left[h^{-1}(b)-a\right]
$$

corresponding to Figures 3 and 4 respectively.

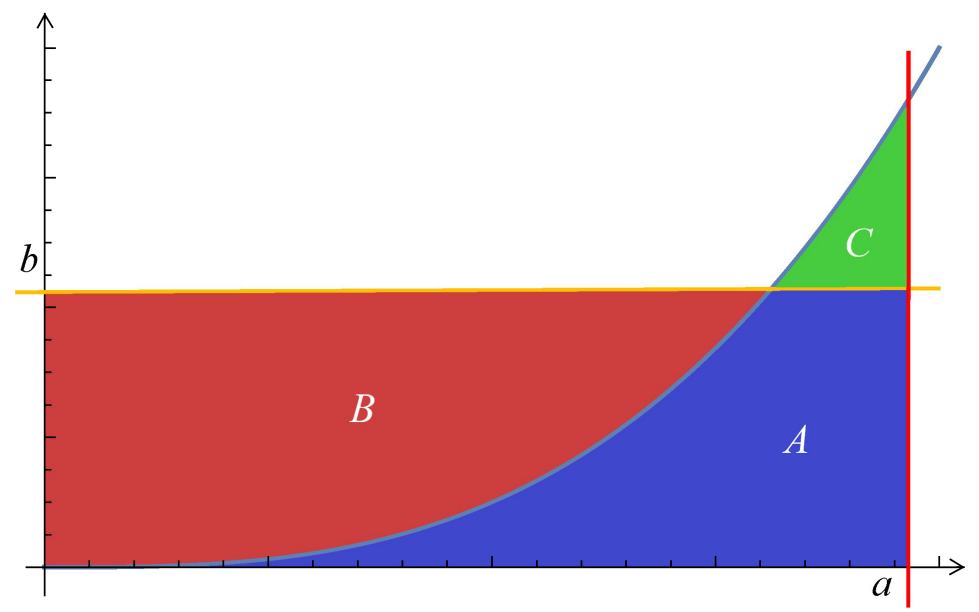

FigurE 3. Geometric interpretation of the double inequality 1.9

Remark 1.7. If $Q$ is a convex function on $J$, then

$$
Q\left(\frac{\tau+\mu}{2}\right) \lesseqgtr \frac{1}{\mu-\tau} \int_{\tau}^{\mu} Q(x) \mathrm{d} x \lesseqgtr \frac{Q(\tau)+Q(\mu)}{2},
$$




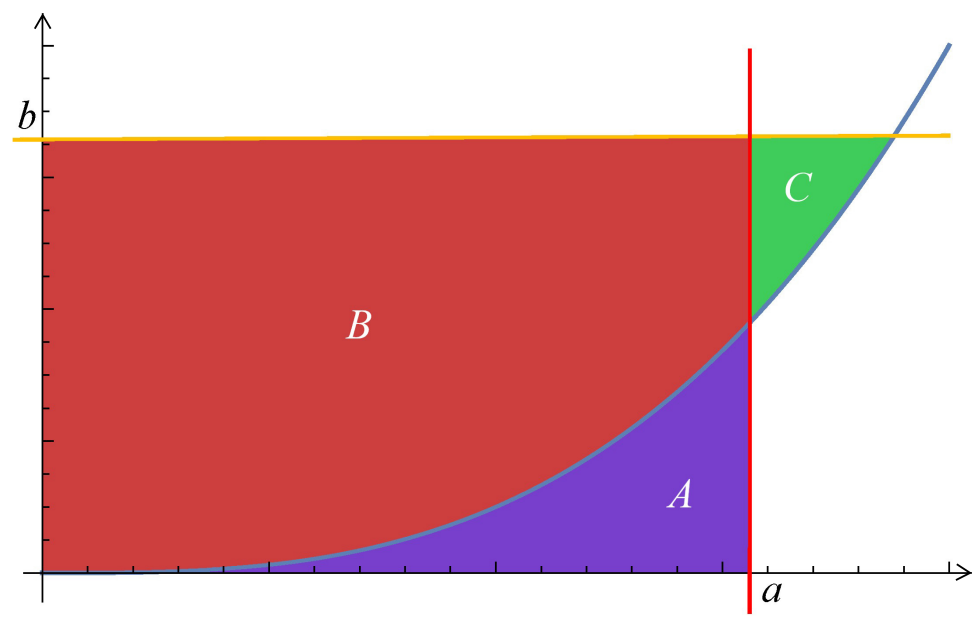

FIgURE 4. Geometric interpretation of the double inequality 1.9

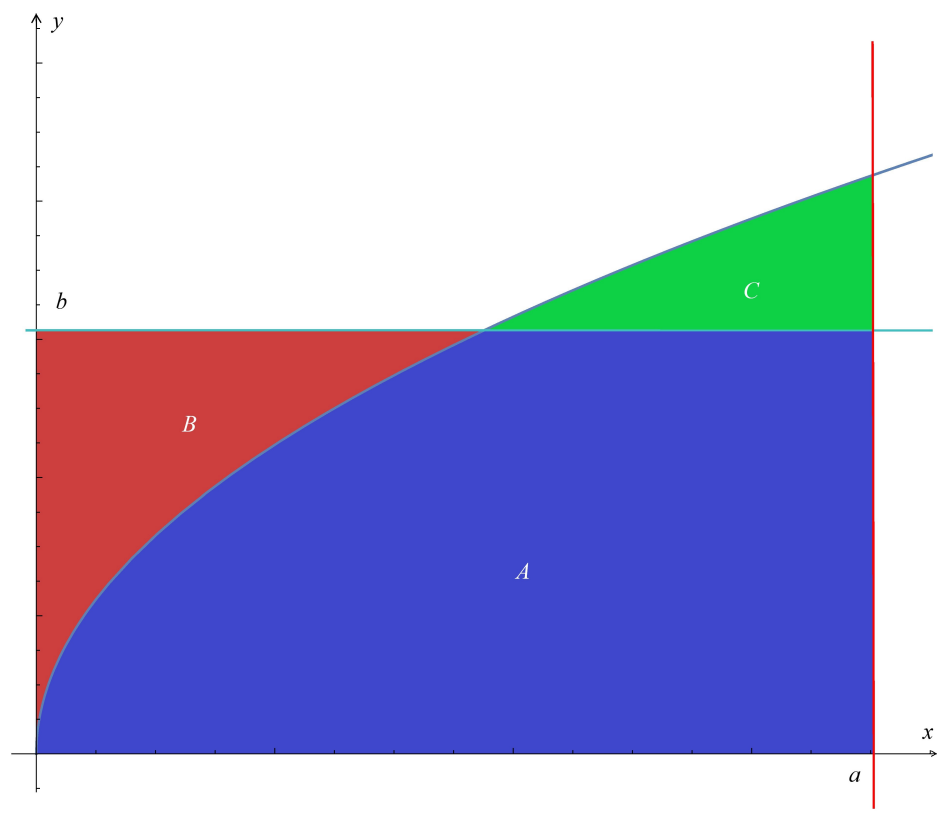

FIGURE 5. Geometric interpretation of the double inequality (1.9)

where $J \subseteq \mathbb{R}$ is a nonempty interval and $\tau, \mu \in J$ with $\tau<\mu$. The double inequality $(1.13)$ is called Hermite-Hadamard's integral inequality for convex functions [7, 37, 44. When $a>h^{-1}(b)$, as showed in Figures 3 and 5 , and $h^{\prime}(x)$ is strictly increasing, that is, the function $h(x)$ is convex, as showed in Figures 3 


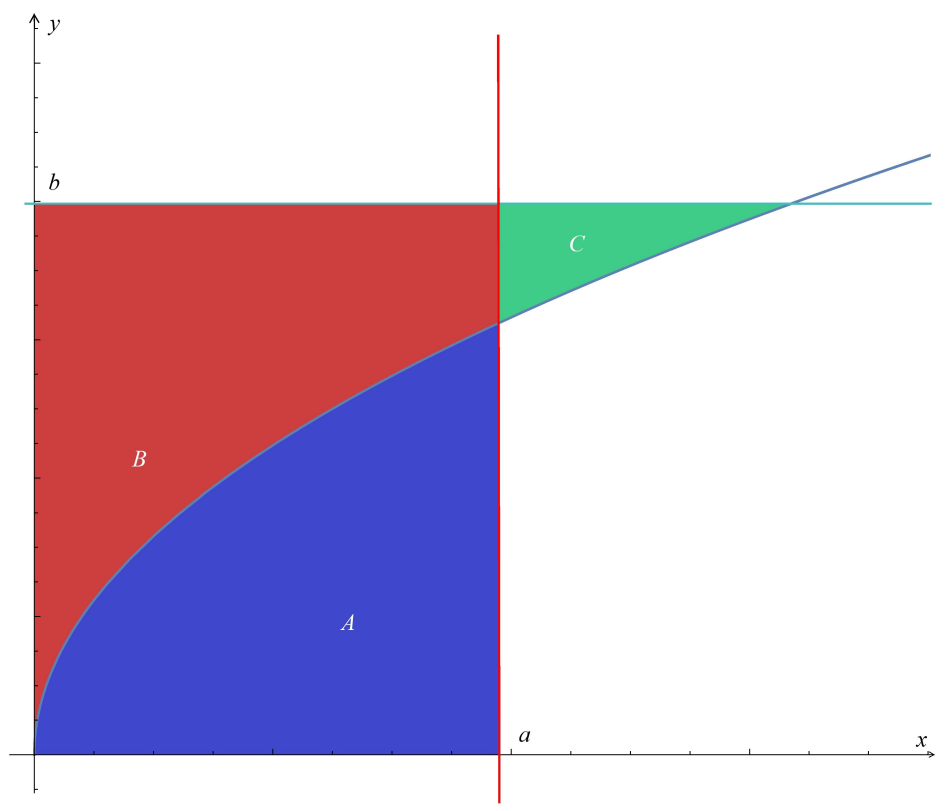

FIGURE 6. Geometric interpretation of the double inequality 1.9 p

and 4 applying the double inequality 1.13 yields

$$
h\left(\frac{a+h^{-1}(b)}{2}\right) \leq \frac{\int_{h^{-1}(b)}^{a} h(x) \mathrm{d} x}{a-h^{-1}(b)} \leq \frac{h(a)+b}{2} .
$$

Substituting this into the third line in 1.10 gives

$$
\int_{0}^{a} h(x) \mathrm{d} x+\int_{0}^{b} h^{-1}(x) \mathrm{d} x \leq a b+\frac{h(a)-b}{2}\left[a-h^{-1}(b)\right]
$$

and

$$
\begin{aligned}
\int_{0}^{a} h(x) \mathrm{d} x+ & \int_{0}^{b} h^{-1}(x) \mathrm{d} x \geq b h^{-1}(b)+h\left(\frac{a+h^{-1}(b)}{2}\right)\left[a-h^{-1}(b)\right] \\
& =a b+\left[h\left(\frac{a+h^{-1}(b)}{2}\right)-b\right]\left[a-h^{-1}(b)\right] .
\end{aligned}
$$

Equivalently speaking, it follows that the area $C$ satisfies

$$
\left[h\left(\frac{a+h^{-1}(b)}{2}\right)-b\right]\left[a-h^{-1}(b)\right] \leq C \leq \frac{h(a)-b}{2}\left[a-h^{-1}(b)\right] .
$$

Similarly, we can discuss other cases, corresponding to Figures 5 and 6 , that the derivative $h^{\prime}(x)$ is strictly decreasing.

Remark 1.8. Mercer has applied and employed the double inequality 1.9 in the paper [23] and in the Undergraduate Texts in Mathematics [24]. 
1.3. Refinements of Young's integral inequality via Hermite-Hadamard's and Čebyšev's integral inequalities. In 2009 and 2010, among other things, Jakšetić and Pečarić refined Young's integral inequality (1.1) and Hoorfar-Qi's double inequality $(1.9)$ in [19, 20].

Theorem 1.3 ([19, Theorem 2.1] and [20, Theorem 2.3]). Let $h(x)$ be a differentiable and strictly increasing function on $[0, c]$ for $c>0, h(0)=0, a \in[0, c]$, $b \in[0, h(c)]$, and $h^{-1}$ be the inverse function of $h$. Denote

$$
\alpha=\min \left\{a, h^{-1}(b)\right\} \quad \text { and } \beta=\max \left\{a, h^{-1}(b)\right\} .
$$

(1) If $h^{\prime}(x)$ is increasing on $[\alpha, \beta]$ and $b<h(a)$, or if $h^{\prime}(x)$ is decreasing on $[\alpha, \beta]$ and $b>h(a)$, then

$$
\begin{aligned}
{\left[a-h^{-1}(b)\right]\left[h\left(\frac{a+h^{-1}(b)}{2}\right)-b\right] } & \leq \int_{0}^{a} h(x) \mathrm{d} x+\int_{0}^{b} h^{-1}(x) \mathrm{d} x-a b \\
& \leq \frac{1}{2}\left[a-h^{-1}(b)\right][h(a)-b] .
\end{aligned}
$$

(2) If $h^{\prime}(x)$ is increasing on $[\alpha, \beta]$ and $b>h(a)$, or if $h^{\prime}(x)$ is decreasing on $[\alpha, \beta]$ and $b<h(a)$, then the inequality (1.15) is reversed.

(3) The equality in 1.15) is valid if and only if $h(x)=\lambda x$ for $\lambda>0$ or $b=h(a)$.

Proof. This is the outline of proofs of [19, Theorem 2.1] and [20, Theorem 2.3].

From the third line in 1.10 , it follows that

$$
\int_{0}^{a} h(x) \mathrm{d} x+\int_{0}^{b} h^{-1}(x) \mathrm{d} x-a b=b\left[h^{-1}(b)-a\right]+\int_{h^{-1}(b)}^{a} h(x) \mathrm{d} x .
$$

Considering monotonicity of $h^{\prime}(x)$ and applying the double inequality 1.13$)$ to the integrand in the last term of (1.16), we can derive the double inequality (1.15).

The last term in 1.10$)$ can be rewritten as

$$
\begin{aligned}
\int_{h^{-1}(b)}^{a}[h(x)-b] \mathrm{d} x=\int_{h^{-1}(b)}^{a}\left[h(x)-h\left(h^{-1}(b)\right)\right] \mathrm{d} x \\
=\int_{h^{-1}(b)}^{a} \int_{h^{-1}(b)}^{x} h^{\prime}(u) \mathrm{d} u \mathrm{~d} x=\int_{h^{-1}(b)}^{a}(a-u) h^{\prime}(u) \mathrm{d} u .
\end{aligned}
$$

Let $f, g:[\mu, \nu] \rightarrow \mathbb{R}$ be integrable functions satisfying that they are both increasing or both decreasing. Then

$$
\int_{\mu}^{\nu} f(x) \mathrm{d} x \int_{\mu}^{\nu} g(x) \mathrm{d} x \leq(\nu-\mu) \int_{\mu}^{\nu} f(x) g(x) \mathrm{d} x .
$$

If one of the function $f$ or $g$ is nonincreasing and the other nondecreasing, then the inequality in $(1.18)$ is reversed. The inequality $(1.18)$ is called Cebyšev's integral inequality in the literature [26, Chapter IX] and [35, 38. Applying $[1.18$ to the last term in 1.17 leads to the right hand side of the inequality 1.15 . The proof of Theorem 1.3 is complete.

Remark 1.9. The double inequality 1.15 can be geometrically interpreted as

$$
\left[a-h^{-1}(b)\right]\left[h\left(\frac{a+h^{-1}(b)}{2}\right)-b\right] \leq C \leq \frac{1}{2}\left[a-h^{-1}(b)\right][h(a)-b],
$$

where $C$ denotes the area showed in Figures 1 to 6 . 
1.4. Refinements of Young's integral inequality via Jensen's discrete and integral inequalities. In [20, Theorem 2.6], Jensen's discrete and integral inequalities were employed to establish the following inequalities which refine Young's integral inequality (1.1) and Hoorfar-Qi's double inequality (1.9).

Theorem 1.4 ([20, Theorem 2.6]). Let $h(x)$ be a differentiable and strictly increasing function on $[0, c]$ for $c>0$ and let $h^{-1}$ be the inverse function of $h$. If $h(0)=0$, $a \in[0, c], b \in[0, h(c)]$, and $h^{\prime}(x)$ is convex on $[\alpha, \beta]$, then

$$
\begin{aligned}
\frac{\left[a-h^{-1}(b)\right]^{2}}{2} h^{\prime}\left(\frac{a+2 h^{-1}(b)}{3}\right) & \leq \int_{0}^{a} h(x) \mathrm{d} x+\int_{0}^{b} h^{-1}(x) \mathrm{d} x-a b \\
& \leq \frac{\left[a-h^{-1}(b)\right]^{2}}{3}\left[\frac{h^{\prime}(a)}{2}+h^{\prime}\left(h^{-1}(b)\right)\right] .
\end{aligned}
$$

If $h^{\prime}(x)$ is concave, then the double inequality 1.19 is reversed.

Proof. This is the outline of the proof of [20, Theorem 2.6].

Changing the variable of the last term in (1.17) results in

$$
\int_{h^{-1}(b)}^{a}(a-u) h^{\prime}(u) \mathrm{d} u=\int_{0}^{1}\left[a-h^{-1}(b)\right]^{2}(1-x) h^{\prime}\left(x a+(1-x) h^{-1}(b)\right) \mathrm{d} x .
$$

If $f$ is a convex function on an interval $I \subseteq \mathbb{R}$ and if $n \geq 2$ and $x_{k} \in I$ for $1 \leq k \leq n$, then

$$
f\left(\frac{1}{\sum_{k=1}^{n} p_{k}} \sum_{k=1}^{n} p_{k} x_{k}\right) \leq \frac{1}{\sum_{k=1}^{n} p_{k}} \sum_{k=1}^{n} p_{k} f\left(x_{k}\right),
$$

where $p_{k}>0$ for $1 \leq k \leq n$. If $f$ is concave, the inequality (1.21) is reversed. The inequality (1.21) is called Jensen's discrete inequality for convex functions in the literature [25, Section 1.4] and [26, Chapter I]. Applying (1.21) to the third factor in the integrand of the right hand side in 1.20 arrives at the right inequality in 1.19 ).

Let $\phi$ be a convex function on $[\mu, \nu], f \in L_{1}(\mu, \nu)$, and $\sigma$ be a non-negative measure. Then

$$
\phi\left(\frac{\int_{\mu}^{\nu} f(x) \mathrm{d} \sigma}{\int_{\mu}^{\nu} \mathrm{d} \sigma}\right) \leq \frac{\int_{\mu}^{\nu} \phi(f(x)) \mathrm{d} \sigma}{\int_{\mu}^{\nu} \mathrm{d} \sigma} .
$$

If $\phi$ is a concave function, then the inequality 1.22 is reversed. The inequality 1.22 is called Jensen's integral inequality for convex functions in the literature [26, p. 10, (7.15)]. Applying (1.22 yields

$$
\begin{aligned}
\int_{h^{-1}(b)}^{a}(a-x) h^{\prime}(x) \mathrm{d} x & \geq \frac{\left[1-h^{-1}(b)\right]^{2}}{2} h^{\prime}\left(\frac{\int_{h^{-1}(b)}^{a}(a-x) x \mathrm{~d} x}{\int_{h^{-1}(b)}^{a}(a-x) \mathrm{d} x}\right) \\
& =\frac{\left[1-h^{-1}(b)\right]^{2}}{2} h^{\prime}\left(\frac{a+2 h^{-1}(b)}{3}\right) .
\end{aligned}
$$

The proof of Theorem 1.4 is complete.

Remark 1.10. The double inequality 1.19 can be geometrically interpreted as

$$
\frac{\left[a-h^{-1}(b)\right]^{2}}{2} h^{\prime}\left(\frac{a+2 h^{-1}(b)}{3}\right) \leq C \leq \frac{\left[a-h^{-1}(b)\right]^{2}}{3}\left[\frac{h^{\prime}(a)}{2}+h^{\prime}\left(h^{-1}(b)\right)\right],
$$

where $C$ denotes the area showed in Figures 1 to 6 . 
1.5. Refinements of Young's integral inequality via Hölder's integral inequality. In [20, Theorem 2.1], Hölder's integral inequality was utilized to present the following inequalities, which refine Young's integral inequality (1.1) and HoorfarQi's double inequality (1.9), in terms of norms.

Theorem 1.5 (20, Theorem 2.1]). Let $h(x)$ be a differentiable and strictly increasing function on $[0, c]$ for $c>0$ and let $h^{-1}$ be the inverse function of $h$. If $h(0)=0$, $a \in[0, c], b \in[0, h(c)]$, and $h^{\prime}(x)$ is almost everywhere continuous with respect to Lebesgue measure on $[\alpha, \beta]$, then the double inequality

$$
C_{u}\left\|h^{\prime}\right\|_{v} \leq \int_{0}^{a} h(x) \mathrm{d} x+\int_{0}^{b} h^{-1}(x) \mathrm{d} x-a b \leq C_{p}\left\|h^{\prime}\right\|_{q}
$$

is valid for all $u, v$ and $p, q$ satisfying

(1) $\frac{1}{u}+\frac{1}{v}=1$ for $u, v \in(-\infty, 0) \cup(0,1)$, or $(u, v)=(1,-\infty)$, or $(u, v)=$ $(-\infty, 1)$

(2) $\frac{1}{p}+\frac{1}{q}=1$ for $1<p, q<\infty$, or $(p, q)=(+\infty, 1)$, or $(p, q)=(1,+\infty)$;

where

$$
C_{r}= \begin{cases}{\left[\frac{\left|a-h^{-1}(b)\right|^{r+1}}{r+1}\right]^{1 / r},} & r \neq 0, \pm \infty ; \\ \left|a-h^{-1}(b)\right|, & r=+\infty ; \\ 0, & r=-\infty\end{cases}
$$

and

$$
\left\|h^{\prime}\right\|_{r}= \begin{cases}{\left[\int_{\alpha}^{\beta}\left[h^{\prime}(t)\right]^{r} \mathrm{~d} t\right]^{1 / r},} & r \neq 0, \pm \infty \\ \sup \left\{h^{\prime}(t), t \in[\alpha, \beta]\right\}, & r=+\infty \\ \inf \left\{h^{\prime}(t), t \in[\alpha, \beta]\right\}, & r=-\infty\end{cases}
$$

Proof. This is the outline of the proof of [20, Theorem 2.1].

Let $\frac{1}{p}+\frac{1}{q}=1$ with $p>0$ and $p \neq 1$, let $f$ and $g$ be real functions on $[\mu, \nu]$, and let $|f|^{p}$ and $|g|^{q}$ be integrable on $[\mu, \nu]$.

(1) If $p>1$, then

$$
\int_{\mu}^{\nu}|f(x) g(x)| \mathrm{d} x \leq\left[\int_{\mu}^{\nu}|f(x)|^{p} \mathrm{~d} x\right]^{1 / p}\left[\int_{\mu}^{\nu}|g(x)|^{q} \mathrm{~d} x\right]^{1 / q} .
$$

The equality in (1.24) holds if and only if $A|f(x)|^{p}=B|g(x)|^{q}$ almost everywhere for two constants $A$ and $B$.

(2) If $0<p<1$, then the inequality (1.24) is reversed.

The inequality (1.24) is called Hölder's integral inequality in the lierature 26, Chapter $\mathrm{V}]$ and $[43,47,48$.

From (1.17), it follows that,

(1) by a property of definite integrals, we have

$$
\begin{aligned}
& \int_{h^{-1}(b)}^{a}(a-u) h^{\prime}(u) \mathrm{d} u=\int_{\beta}^{\alpha}|a-u| h^{\prime}(u) \mathrm{d} u \\
& \leq\left|h^{-1}(b)-a\right| \int_{\beta}^{\alpha} h^{\prime}(u) \mathrm{d} u=C_{\infty}\left\|h^{\prime}\right\|_{1} ;
\end{aligned}
$$


(2) by a property of definite integrals, we have

$$
\int_{h^{-1}(b)}^{a}(a-u) h^{\prime}(u) \mathrm{d} u=\int_{\beta}^{\alpha}|a-u| h^{\prime}(u) \mathrm{d} u \leq C_{1}\left\|h^{\prime}\right\|_{\infty} ;
$$

(3) by Hölder's integral inequality 1.24 , we have

$$
\begin{aligned}
\int_{h^{-1}(b)}^{a}(a-u) h^{\prime}(u) \mathrm{d} u & =\int_{\beta}^{\alpha}|a-u| h^{\prime}(u) \mathrm{d} u \\
\leq & \left(\int_{\beta}^{\alpha}|a-u|^{q} \mathrm{~d} u\right)^{1 / q}\left(\int_{\beta}^{\alpha}\left[h^{\prime}(u)\right]^{p} \mathrm{~d} u\right)^{1 / p}=C_{q}\left\|h^{\prime}\right\|_{p} .
\end{aligned}
$$

The rest proofs are straightforward. The proofs of the double inequality $(1.23)$ and Theorem 1.5 are thus complete.

Remark 1.11. The double inequality 1.23 can be geometrically interpreted as

$$
C_{u}\left\|h^{\prime}\right\|_{v} \leq C \leq C_{p}\left\|h^{\prime}\right\|_{q}
$$

where $C$ denotes the area showed in Figures 1 to 6.

1.6. Refinements of Young's integral inequality via Taylor's mean value theorem of Lagrange's type remainder. In [49, Theorem 3.1], making use of Taylor's mean value theorem of Lagrange's type remainder, Wang, Guo, and Qi refined the above inequalities of Young's type via higher order derivatives.

Theorem 1.6 (49, Theorem 3.1]). Let $h(0)=0$ and $h(x)$ be strictly increasing on $[0, c]$ for $c>0$, let $h^{(n)}(x)$ for $n \geq 0$ be continuous on $[0, c]$, let $h^{(n+1)}(x)$ be finite and strictly monotonic on $(0, c)$, and let $h^{-1}$ be the inverse function of $h$. For $a \in[0, c]$ and $b \in[0, h(c)]$,

(1) if $b<h(a)$, then

$$
\begin{aligned}
& \sum_{k=1}^{n} h^{(k)}\left(h^{-1}(b)\right) \frac{\left[a-h^{-1}(b)\right]^{k+1}}{(k+1) !}+m_{n}(a, b) \frac{\left[a-h^{-1}(b)\right]^{n+2}}{(n+2) !} \\
\leq & \int_{0}^{a} h(x) \mathrm{d} x+\int_{0}^{b} h^{-1}(x) \mathrm{d} x-a b \\
\leq & \sum_{k=1}^{n} h^{(k)}\left(h^{-1}(b)\right) \frac{\left[a-h^{-1}(b)\right]^{k+1}}{(k+1) !}+M_{n}(a, b) \frac{\left[a-h^{-1}(b)\right]^{n+2}}{(n+2) !},
\end{aligned}
$$

where

$$
m_{n}(a, b)=\min \left\{h^{(n+1)}\left(h^{-1}(b)\right), h^{(n+1)}(a)\right\}
$$

and

$$
M_{n}(a, b)=\max \left\{h^{(n+1)}\left(h^{-1}(b)\right), h^{(n+1)}(a)\right\} ;
$$

(2) if $b>h(a)$, then

(a) when $n=2 \ell$ for $\ell \geq 0$, the double inequality 1.25 is valid; 
(b) when $n=2 \ell+1$ for $\ell \geq 0$, we have

$$
\begin{aligned}
& \sum_{k=1}^{n} h^{(k)}\left(h^{-1}(b)\right) \frac{\left[a-h^{-1}(b)\right]^{k+1}}{(k+1) !}-M_{n}(a, b) \frac{\left[a-h^{-1}(b)\right]^{n+2}}{(n+2) !} \\
\leq & \int_{0}^{a} h(x) \mathrm{d} x+\int_{0}^{b} h^{-1}(x) \mathrm{d} x-a b \\
\leq & \sum_{k=1}^{n} h^{(k)}\left(h^{-1}(b)\right) \frac{\left[a-h^{-1}(b)\right]^{k+1}}{(k+1) !}-m_{n}(a, b) \frac{\left[a-h^{-1}(b)\right]^{n+2}}{(n+2) !}
\end{aligned}
$$

(3) if, and only if, $b=h(a)$, those equalities in 1.25 and 1.26 hold.

Proof. This is the outline of the proof of [49, Theorem 3.1].

Let $f(x)$ be a function having finite $n$th derivative $f^{(n)}(x)$ everywhere in an open interval $(\mu, \nu)$ and assume that $f^{(n-1)}(x)$ is continuous on the closed interval $[\mu, \nu]$. Then, for a fixed point $x_{0} \in[\mu, \nu]$ and every $x \in[\mu, \nu]$ with $x \neq x_{0}$, there exists a point $x_{1}$ interior to the interval jointing $x$ and $x_{0}$ such that

$$
f(x)=f\left(x_{0}\right)+\sum_{k=1}^{n-1} \frac{f^{(k)}\left(x_{0}\right)}{k !}\left(x-x_{0}\right)^{k}+\frac{f^{(n)}\left(x_{1}\right)}{n !}\left(x-x_{0}\right)^{n} .
$$

The formula 1.27 is called Taylor's mean value theorem of Lagrange's type remainder in the literature [6, p. 113, Theorem 5.19]. Applying (1.27) in the last term of 1.10 reveals

$$
\begin{aligned}
\int_{h^{-1}(b)}^{a}[h(x)-b] \mathrm{d} x= & \int_{h^{-1}(b)}^{a}\left[h(x)-h\left(h^{-1}(b)\right)\right] \mathrm{d} x \\
= & \sum_{k=1}^{n} \frac{h^{(k)}\left(h^{-1}(b)\right)}{k !} \int_{h^{-1}(b)}^{a}\left[x-h^{-1}(b)\right]^{k} \mathrm{~d} x \\
& +\frac{1}{(n+1) !} \int_{h^{-1}(b)}^{a} h^{(n+1)}(\xi)\left[x-h^{-1}(b)\right]^{n+1} \mathrm{~d} x \\
= & \sum_{k=1}^{n} h^{(k)}\left(h^{-1}(b)\right) \frac{\left[a-h^{-1}(b)\right]^{k+1}}{(k+1) !} \\
& +\int_{h^{-1}(b)}^{a} h^{(n+1)}(\xi) \frac{\left[x-h^{-1}(b)\right]^{n+1}}{(n+1) !} \mathrm{d} x,
\end{aligned}
$$

where $\xi$ is a point interior to the interval jointing $x$ and $h^{-1}(b)$. The rest proofs are straightforward discussions on various cases of the factor $h^{(n+1)}(\xi)$. The proof of Theorem 1.6 is complete.

Remark 1.12. The double inequalities 1.25 and 1.26 can be geometrically interpreted as

$$
\begin{aligned}
& \sum_{k=1}^{n} h^{(k)}\left(h^{-1}(b)\right) \frac{\left[a-h^{-1}(b)\right]^{k+1}}{(k+1) !}+m_{n}(a, b) \frac{\left[a-h^{-1}(b)\right]^{n+2}}{(n+2) !} \leq C \\
& \leq \sum_{k=1}^{n} h^{(k)}\left(h^{-1}(b)\right) \frac{\left[a-h^{-1}(b)\right]^{k+1}}{(k+1) !}+M_{n}(a, b) \frac{\left[a-h^{-1}(b)\right]^{n+2}}{(n+2) !}
\end{aligned}
$$


and

$$
\begin{aligned}
& \sum_{k=1}^{n} h^{(k)}\left(h^{-1}(b)\right) \frac{\left[a-h^{-1}(b)\right]^{k+1}}{(k+1) !}-M_{n}(a, b) \frac{\left[a-h^{-1}(b)\right]^{n+2}}{(n+2) !} \leq C \\
& \leq \sum_{k=1}^{n} h^{(k)}\left(h^{-1}(b)\right) \frac{\left[a-h^{-1}(b)\right]^{k+1}}{(k+1) !}-m_{n}(a, b) \frac{\left[a-h^{-1}(b)\right]^{n+2}}{(n+2) !}
\end{aligned}
$$

where $C$ denotes the area showed in Figures 1 to 6 .

1.7. Refinements of Young's integral inequality via Taylor's mean value theorem of Cauchy's type remainder and Hölder's integral inequality. In [49, Theorem 3.2], employing Taylor's mean value theorem of Cauchy's type remainder and Hölder's integral inequality, Wang, Guo, and Qi refined the above inequalities of Young's type via norms of higher order derivatives.

Theorem 1.7 ([49, Theorem 3.2]). Let $n \geq 0$ and $h(x) \in C^{n+1}[0, c]$ such that $h(0)=0, h^{(n+1)}(x) \geq 0$ on $[\alpha, \beta]$, and $h(x)$ is strictly increasing on $[0, c]$ for $c>0$, let $h^{-1}$ be the inverse function of $h$, and let $a \in[0, c]$ and $b \in[0, h(c)]$. Then

(1) when $b>h(a)$ and $n=2 \ell$ for $\ell \geq 0$ or when $b<h(a)$, we have

$$
\begin{aligned}
\frac{C_{u, n}}{(n+1) !}\left\|h^{(n+1)}\right\|_{v} \leq & \int_{0}^{a} h(x) \mathrm{d} x+\int_{0}^{b} h^{-1}(x) \mathrm{d} x-a b \\
& -\sum_{k=1}^{n} h^{(k)}\left(h^{-1}(b)\right) \frac{\left[a-h^{-1}(b)\right]^{k+1}}{(k+1) !} \\
\leq & \frac{C_{p, n}}{(n+1) !}\left\|h^{(n+1)}\right\|_{q}
\end{aligned}
$$

(2) when $b>h(a)$ and $n=2 \ell+1$ for $\ell \geq 0$, we have

$$
\begin{aligned}
-\frac{C_{p, n}}{(n+1) !}\left\|h^{(n+1)}\right\|_{q} \leq & \int_{0}^{a} h(x) \mathrm{d} x+\int_{0}^{b} h^{-1}(x) \mathrm{d} x-a b \\
& -\sum_{k=1}^{n} h^{(k)}\left(h^{-1}(b)\right) \frac{\left[a-h^{-1}(b)\right]^{k+1}}{(k+1) !} \\
\leq & -\frac{C_{u, n}}{(n+1) !}\left\|h^{(n+1)}\right\|_{v} ;
\end{aligned}
$$

where $\alpha, \beta$ are defined as in 1.14,

$$
\begin{gathered}
C_{r, n}= \begin{cases}{\left[\frac{\left|a-h^{-1}(b)\right|^{r(n+1)+1}}{r(n+1)+1}\right]^{1 / r},} & r \neq 0, \pm \infty ; \\
\left|a-h^{-1}(b)\right|^{n+1}, & r=+\infty ; \\
0, & r=-\infty,\end{cases} \\
\left\|h^{(n+1)}\right\|_{r}= \begin{cases}{\left[\int_{\alpha}^{\beta}\left[h^{(n+1)}(t)\right]^{r} \mathrm{~d} t\right]^{1 / r},} & r \neq 0, \pm \infty ; \\
\sup \left\{h^{(n+1)}(t), t \in[\alpha, \beta]\right\}, & r=+\infty ; \\
\inf \left\{h^{(n+1)}(t), t \in[\alpha, \beta]\right\}, & r=-\infty,\end{cases}
\end{gathered}
$$

and $u, v, p, q$ satisfy 
(1) $u<1$ and $u \neq 0$ with $\frac{1}{u}+\frac{1}{v}=1$, or $(u, v)=(-\infty, 1)$, or $(u, v)=(1,-\infty)$;

(2) $1<p, q<\infty$ with $\frac{1}{p}+\frac{1}{q}=1$, or $(p, q)=(+\infty, 1)$, or $(p, q)=(1,+\infty)$.

Proof. This is the outline of the proof of [49, Theorem 3.2].

If $f(x) \in C^{n+1}[\mu, \nu]$ and $x_{0} \in[\mu, \nu]$, then

$$
f(x)=\sum_{k=0}^{n} \frac{f^{(k)}\left(x_{0}\right)}{k !}\left(x-x_{0}\right)^{k}+\frac{1}{n !} \int_{x_{0}}^{x}(x-t)^{n} f^{(n+1)}(t) \mathrm{d} t .
$$

The formula 1.28 is called Taylor's mean value theorem of Cauchy's type remainder in the literature [5, p. 279, Theorem 7.6] and [27, p. 6, 1.4.37]. Applying the formula 1.28 to the integrand in the last term of 1.10 yields

$$
\begin{aligned}
\int_{h^{-1}(b)}^{a}[h(x)-b] \mathrm{d} x= & \int_{h^{-1}(b)}^{a}\left[h(x)-h\left(h^{-1}(b)\right)\right] \mathrm{d} x \\
= & \sum_{k=1}^{n} h^{(k)}\left(h^{-1}(b)\right) \frac{\left[a-h^{-1}(b)\right]^{k+1}}{(k+1) !} \\
& +\int_{h^{-1}(b)}^{a} \frac{1}{n !} \int_{h^{-1}(b)}^{x}(x-t)^{n} h^{(n+1)}(t) \mathrm{d} t \mathrm{~d} x \\
= & \sum_{k=1}^{n} h^{(k)}\left(h^{-1}(b)\right) \frac{\left[a-h^{-1}(b)\right]^{k+1}}{(k+1) !} \\
& +\int_{h^{-1}(b)}^{a} \frac{1}{n !} \int_{t}^{a}(x-t)^{n} h^{(n+1)}(t) \mathrm{d} x \mathrm{~d} t \\
= & \sum_{k=1}^{n} h^{(k)}\left(h^{-1}(b)\right) \frac{\left[a-h^{-1}(b)\right]^{k+1}}{(k+1) !} \\
& +\frac{1}{(n+1) !} \int_{h^{-1}(b)}^{a} \\
= & \sum_{k=1}^{n} h^{(k)}\left(h^{-1}(b)\right) \frac{(a-t)^{n+1} h^{(n+1)}(t) \mathrm{d} t}{(k+1) !} \\
& +\left\{\begin{array}{l}
\frac{1}{(n+1) !} \int_{\alpha}^{\beta}\left(a-\left.t\right|^{n+1} h^{(n+1)}(t) \mathrm{d} t, \quad b<h(a) ;\right. \\
\frac{(-1)^{n}}{(n+1) !} \int_{\alpha}^{\beta}|a-t|^{n+1} h^{(n+1)}(t) \mathrm{d} t, \quad b>h(a) .
\end{array}\right.
\end{aligned}
$$

Discussing and making use of Hölder's integral inequality $(1.24)$ as in the proof of Theorem 1.5, we can complete the proof of Theorem 1.7 .

Remark 1.13. Two double inequalities in Theorem 1.7 can be geometrically interpreted as

$$
\begin{aligned}
\frac{C_{u, n}}{(n+1) !}\left\|h^{(n+1)}\right\|_{v} & \leq C-\sum_{k=1}^{n} h^{(k)}\left(h^{-1}(b)\right) \frac{\left[a-h^{-1}(b)\right]^{k+1}}{(k+1) !} \\
& \leq \frac{C_{p, n}}{(n+1) !}\left\|h^{(n+1)}\right\|_{q}
\end{aligned}
$$


and

$$
\begin{aligned}
-\frac{C_{p, n}}{(n+1) !}\left\|h^{(n+1)}\right\|_{q} & \leq C-\sum_{k=1}^{n} h^{(k)}\left(h^{-1}(b)\right) \frac{\left[a-h^{-1}(b)\right]^{k+1}}{(k+1) !} \\
& \leq-\frac{C_{u, n}}{(n+1) !}\left\|h^{(n+1)}\right\|_{v},
\end{aligned}
$$

where $C$ denotes the area showed in Figures 1 to 6 .

1.8. Refinements of Young's integral inequality via Taylor's mean value theorem of Cauchy's type remainder and Čebyšev's integral inequality.

Theorem 1.8 ([49, Theorem 3.3]). Let $n \geq 0$ and $h(x) \in C^{n+1}[0, c]$ such that $h(0)=0$ and $h(x)$ is strictly increasing on $[0, c]$ for $c>0$, let $h^{-1}$ be the inverse function of $h$, let $a \in[0, c]$ and $b \in[0, h(c)]$, and let $\ell \geq 0$ be an integer. Then

(1) when

(a) either $h(a)>b$ and $h^{(n+1)}(x)$ is increasing on $[\alpha, \beta]$;

(b) or $h(a)<b, h^{(n+1)}(x)$ is increasing on $[\alpha, \beta]$, and $n=2 \ell+1$;

(c) or $h(a)<b, h^{(n+1)}(x)$ is decreasing on $[\alpha, \beta]$, and $n=2 \ell$;

the inequality

$$
\begin{aligned}
& \int_{0}^{a} h(x) \mathrm{d} x+\int_{0}^{b} h^{-1}(x) \mathrm{d} x-a b \\
- & \sum_{k=1}^{n} h^{(k)}\left(h^{-1}(b)\right) \frac{\left[a-h^{-1}(b)\right]^{k+1}}{(k+1) !} \\
\leq & \frac{\left[a-h^{-1}(b)\right]^{n+1}}{(n+2) !}\left[h^{(n)}(a)-h^{(n)}\left(h^{-1}(b)\right)\right]
\end{aligned}
$$

is valid;

(2) when
(a) either $h(a)>b$ and $h^{(n+1)}(x)$ is decreasing on $[\alpha, \beta]$;
(b) or $h(a)<b, h^{(n+1)}(x)$ is increasing on $[\alpha, \beta]$, and $n=2 \ell$;
(c) or $h(a)<b, h^{(n+1)}(x)$ is decreasing on $[\alpha, \beta]$, and $n=2 \ell+1$;
the inequality 1.29 is reversed;

where $\alpha, \beta$ are defined as in 1.14.

Proof. This is the outline of the proof of [49, Theorem 3.3].

This follows from applying the formula $(1.28)$ as in the proof of Theorem 1.7 and applying Čebyšev's integral inequality 1.18 to the integral

$$
\int_{h^{-1}(b)}^{a}(a-t)^{n+1} h^{(n+1)}(t) \mathrm{d} t
$$

in the proof of Theorem 1.7. The proof of Theorem 1.8 is complete.

Remark 1.14 . The inequality $(1.29)$ can be geometrically interpreted as

$$
\begin{aligned}
& C-\sum_{k=1}^{n} h^{(k)}\left(h^{-1}(b)\right) \frac{\left[a-h^{-1}(b)\right]^{k+1}}{(k+1) !} \\
& \leq \frac{\left[a-h^{-1}(b)\right]^{n+1}}{(n+2) !}\left[h^{(n)}(a)-h^{(n)}\left(h^{-1}(b)\right)\right],
\end{aligned}
$$


where $C$ denotes the area showed in Figures 1 to 6 .

\subsection{Refinements of Young's integral inequality via Taylor's mean value theorem of Cauchy's type remainder and Jensen's inequalities.}

Theorem 1.9 ([49, Theorem 3.4]). Let $h(x) \in C^{n+1}[0, c]$ such that $h(0)=0$ and $h(x)$ is strictly increasing on $[0, c]$ for $c>0$, let $h^{-1}$ be the inverse function of $h$, and let $a \in[0, c]$ and $b \in[0, h(c)]$. If $h^{(n+1)}(x)$ is convex on $[\alpha, \beta]$, where $\alpha$, $\beta$ are defined as in 1.14, then

(1) when $h(a)>b$ or when $h(a)<b$ and $n=2 \ell$, we have

$$
\begin{aligned}
& \frac{\left[a-h^{-1}(b)\right]^{n+2}}{n+2} h^{(n+1)}\left(\frac{a+(n+2) h^{-1}(b)}{n+3}\right) \\
\leq & \int_{0}^{a} h(x) \mathrm{d} x+\int_{0}^{b} h^{-1}(x) \mathrm{d} x-a b \\
& -\sum_{k=1}^{n} h^{(k)}\left(h^{-1}(b)\right) \frac{\left[a-h^{-1}(b)\right]^{k+1}}{(k+1) !} \\
\leq & {\left[a-h^{-1}(b)\right]^{n+2} \frac{h^{(n+1)}(a)+(n+2) h^{(n+1)}\left(h^{-1}(b)\right)}{(n+3) !} }
\end{aligned}
$$

(2) when $h(a)<b$ and $n=2 \ell+1$, the double inequality 1.31 is reversed;

where $\ell \geq 0$ is an integer. If $h^{(n+1)}(x)$ is concave on $[\alpha, \beta]$, all the above inequalities are reversed for all corresponding cases.

Proof. This is the outline of the proof of [49, Theorem 3.4].

Considering the integral 1.30 and substituting integral variables give

$$
\begin{aligned}
& \int_{h^{-1}(b)}^{a}(a-t)^{n+1} h^{(n+1)}(t) \mathrm{d} t=\left[a-h^{-1}(b)\right]^{n+2} \\
& \times \int_{0}^{1}(1-s)^{n+1} h^{(n+1)}\left(s a+(1-s) h^{-1}(b)\right) \mathrm{d} s .
\end{aligned}
$$

Applying Jensen's inequalities (1.21) and 1.22 to $h^{(n+1)}\left(s a+(1-s) h^{-1}(b)\right)$ in the above equation yield the double inequality (1.31) and its reversed version. The proof of Theorem 1.9 is complete.

Remark 1.15. The double inequality 1.31 can be geometrically interpreted as

$$
\begin{aligned}
& \frac{\left[a-h^{-1}(b)\right]^{n+2}}{n+2} h^{(n+1)}\left(\frac{a+(n+2) h^{-1}(b)}{n+3}\right) \\
& \leq C-\sum_{k=1}^{n} h^{(k)}\left(h^{-1}(b)\right) \frac{\left[a-h^{-1}(b)\right]^{k+1}}{(k+1) !} \\
& \leq\left[a-h^{-1}(b)\right]^{n+2} \frac{h^{(n+1)}(a)+(n+2) h^{(n+1)}\left(h^{-1}(b)\right)}{(n+3) !},
\end{aligned}
$$

where $C$ denotes the area showed in Figures 1 to 6 . 
1.10. Refinements of Young's integral inequality via Taylor's mean value theorem of Cauchy's type remainder and integral inequalities of HermiteHadamard type for the product of two convex functions.

Theorem 1.10 ([49, Theorem 3.5]). Let $n \geq 0$ and $h(x) \in C^{n+1}[0, c]$ such that $h(0)=0$ and $h(x)$ is strictly increasing on $[0, c]$ for $c>0$, let $h^{-1}$ be the inverse function of $h$, let $a \in[0, c]$ and $b \in[0, h(c)]$, and let $h^{(n+1)}(x)$ be nonnegative and convex on $[\alpha, \beta]$, where $\alpha, \beta$ are defined as in $[1.14$. If $h(a)>b$, then

$$
\begin{aligned}
& \frac{\left[a-h^{-1}(b)\right]^{n+2}}{(n+1) !}\left[\frac{1}{2^{n}} h^{(n+1)}\left(\frac{a+h^{-1}(b)}{2}\right)\right. \\
& \left.-\frac{2 h^{(n+1)}(a)+h^{(n+1)}\left(h^{-1}(b)\right)}{6}\right] \\
\leq & \int_{0}^{a} h(x) \mathrm{d} x+\int_{0}^{b} h^{-1}(x) \mathrm{d} x-a b \\
& -\sum_{k=1}^{n} h^{(k)}\left(h^{-1}(b)\right) \frac{\left[a-h^{-1}(b)\right]^{k+1}}{(k+1) !} \\
\leq & \frac{\left[a-h^{-1}(b)\right]^{n+2}}{(n+1) !} \frac{h^{(n+1)}(a)+2 h^{(n+1)}\left(h^{-1}(b)\right)}{6} .
\end{aligned}
$$

If $h(a)<b$ and $n=2 \ell$ for $\ell \geq 0$, then

$$
\begin{aligned}
& \frac{\left[h^{-1}(b)-a\right]^{n+2}}{(n+1) !}\left[\frac{1}{2^{n}} h^{(n+1)}\left(\frac{a+h^{-1}(b)}{2}\right)\right. \\
& \left.-\frac{2 h^{(n+1)}(a)+h^{(n+1)}\left(h^{-1}(b)\right)}{6}\right] \\
\leq & \int_{0}^{a} h(x) \mathrm{d} x+\int_{0}^{b} h^{-1}(x) \mathrm{d} x-a b \\
& -\sum_{k=1}^{n} h^{(k)}\left(h^{-1}(b)\right) \frac{\left[a-h^{-1}(b)\right]^{k+1}}{(k+1) !} \\
\leq & \frac{\left[h^{-1}(b)-a\right]^{n+2}}{(n+1) !} \frac{h^{(n+1)}(a)+2 h^{(n+1)}\left(h^{-1}(b)\right)}{6} .
\end{aligned}
$$

If $a<h^{-1}(b)$ and $n=2 \ell+1$ for $\ell \geq 0$, the double inequality 1.33 is reversed.

Proof. This is the outline of the proof of [49, Theorem 3.5].

Let $f(x)$ and $g(x)$ be nonnegative and convex functions on $[\mu, \nu]$. Then

$$
\begin{aligned}
2 f\left(\frac{\mu+\nu}{2}\right) g\left(\frac{\mu+\nu}{2}\right) & -\frac{1}{6} M(\mu, \nu)-\frac{1}{3} N(\mu, \nu) \\
\leq & \frac{1}{\nu-\mu} \int_{\mu}^{\nu} f(x) g(x) \mathrm{d} x \leq \frac{1}{3} M(\mu, \nu)+\frac{1}{6} N(\mu, \nu),
\end{aligned}
$$

where

$$
M(\mu, \nu)=f(\mu) g(\mu)+f(\nu) g(\nu) \quad \text { and } \quad N(\mu, \nu)=f(\mu) g(\nu)+f(\nu) g(\mu) .
$$


The double inequality 51.34 can be found in [28, 51, 52, 53] and closely related references therein. Applying (1.34) in the integral 1.30 arrives at the double inequalities in 1.32 and 1.33 . The proof of Theorem 1.10 is complete.

Remark 1.16. The double inequalities 1.32 and 1.33 can be geometrically interpreted as

$$
\begin{gathered}
\frac{\left[a-h^{-1}(b)\right]^{n+2}}{(n+1) !}\left[\frac{1}{2^{n}} h^{(n+1)}\left(\frac{a+h^{-1}(b)}{2}\right)-\frac{2 h^{(n+1)}(a)+h^{(n+1)}\left(h^{-1}(b)\right)}{6}\right] \\
\leq C-\sum_{k=1}^{n} h^{(k)}\left(h^{-1}(b)\right) \frac{\left[a-h^{-1}(b)\right]^{k+1}}{(k+1) !} \\
\leq \frac{\left[a-h^{-1}(b)\right]^{n+2}}{(n+1) !} \frac{h^{(n+1)}(a)+2 h^{(n+1)}\left(h^{-1}(b)\right)}{6}
\end{gathered}
$$

and

$$
\begin{gathered}
\frac{\left[h^{-1}(b)-a\right]^{n+2}}{(n+1) !}\left[\frac{1}{2^{n}} h^{(n+1)}\left(\frac{a+h^{-1}(b)}{2}\right)-\frac{2 h^{(n+1)}(a)+h^{(n+1)}\left(h^{-1}(b)\right)}{6}\right] \\
\leq C-\sum_{k=1}^{n} h^{(k)}\left(h^{-1}(b)\right) \frac{\left[a-h^{-1}(b)\right]^{k+1}}{(k+1) !} \\
\leq \frac{\left[h^{-1}(b)-a\right]^{n+2}}{(n+1) !} \frac{h^{(n+1)}(a)+2 h^{(n+1)}\left(h^{-1}(b)\right)}{6},
\end{gathered}
$$

where $C$ denotes the area showed in Figures 1 to 6.

\subsection{Three examples showing refinements of Young's integral inequality.}

1.11.1. First example. In [18, Section 3], the double inequality 1.9 was applied to obtain the estimate

$$
\begin{aligned}
9.000042866 \ldots & =\frac{4 \sqrt[4]{125}}{27}(3-2 \sqrt[4]{5})^{2} \\
& <\int_{0}^{3} \sqrt[4]{x^{4}+1} \mathrm{~d} x+\int_{1}^{3} \sqrt[4]{x^{4}-1} \mathrm{~d} x-9 \\
& <\frac{27}{2 \sqrt[4]{82^{3}}}(3-2 \sqrt[4]{5})^{2} \\
& =9.000042871 \ldots
\end{aligned}
$$

whose gap between the upper and lower bounds is $0.000000005 \ldots$ and which refines a known result

$$
9<\int_{0}^{3} \sqrt[4]{x^{4}+1} \mathrm{~d} x+\int_{1}^{3} \sqrt[4]{x^{4}-1} \mathrm{~d} x<9.0001
$$

In [20, Example 2.5] and [20, Remark 2.7], it was obtained that

$$
9.000042866<\int_{0}^{3} \sqrt[4]{x^{4}+1} \mathrm{~d} x+\int_{1}^{3} \sqrt[4]{x^{4}-1} \mathrm{~d} x<9.000042868880
$$

and

$$
9.000042868058<\int_{0}^{3} \sqrt[4]{x^{4}+1} \mathrm{~d} x+\int_{1}^{3} \sqrt[4]{x^{4}-1} \mathrm{~d} x<9.000042868066
$$


whose gaps between the upper and lower bounds are

$$
0.0000000028 \ldots \text { and } 0.000000000008 \ldots
$$

respectively.

In [19, Example 2.1], it was estimated that

$$
9.00004286765564<\int_{0}^{3} \sqrt[4]{x^{4}+1} \mathrm{~d} x+\int_{1}^{3} \sqrt[4]{x^{4}-1} \mathrm{~d} x<9.00004286805781 .
$$

whose gap between the upper and lower bounds is $0.0000000004021 \ldots$.

In [49, Example 4.1], by virtue of the double inequality (1.31), the above double inequality was refined as

$$
\begin{aligned}
9.0000428983186013 \ldots= & \frac{\left(3-80^{1 / 4}\right)^{3}}{3} \frac{3072(\sqrt[4]{95} \sqrt{2}+3)^{2}}{\left[(\sqrt[4]{95} \sqrt{2}+3)^{4}+256\right]^{7 / 4}} \\
& +9+\frac{8 \times 5^{3 / 4}}{27} \frac{\left(3-80^{1 / 4}\right)^{2}}{2 !} \\
\geq & \int_{0}^{3} \sqrt[4]{x^{4}+1} \mathrm{~d} x+\int_{1}^{3} \sqrt[4]{x^{4}-1} \mathrm{~d} x \\
\geq & \frac{\left(3-80^{1 / 4}\right)^{3}}{4 !}\left(\frac{27}{82^{7 / 4}}+3 \times \frac{4 \sqrt{5}}{729}\right) \\
& +9+\frac{8 \times 5^{3 / 4}}{27} \frac{\left(3-80^{1 / 4}\right)^{2}}{2 !} \\
= & 9.0000428680640760 \ldots
\end{aligned}
$$

whose gap between the upper and lower bounds is $0.00000003025452 \ldots$.

1.11.2. Second example. In [49, Example 4.2], by virtue of the double inequality 1.15, it follows that

$$
\begin{aligned}
0.364469045537996606 \ldots & =\frac{1}{4}+\left(\frac{1}{2}-\frac{1}{\ln 2}\right)\left[\frac{1}{\exp \left[\frac{1}{2}\left(\frac{1}{2}+\frac{1}{\ln 2}\right)\right]}-\frac{1}{2}\right] \\
& \leq \int_{0}^{1 / 2} \frac{1}{e^{1 / x}} \mathrm{~d} x-\int_{0}^{1 / 2} \frac{1}{\ln x} \mathrm{~d} x \\
& \leq \frac{1}{4}+\frac{1}{2}\left(\frac{1}{2}-\frac{1}{\ln 2}\right)\left(\frac{1}{e^{2}}-\frac{1}{2}\right) \\
& =0.421883810040011829 \ldots
\end{aligned}
$$

The gap between the upper and lower bounds in the double inequality 1.35 is $0.057414764502015 \ldots$

1.11.3. Third example. In [49, Example 4.3], by virtue of the double inequality (1.32, we can obtain the estimate

$$
2.044751320 \ldots \leq \int_{0}^{1} e^{t^{2}} \mathrm{~d} t+\int_{0}^{1} \sqrt{\ln (1+t)} \mathrm{d} t \leq 2.060536019 \ldots
$$

The gap between the upper and lower bounds in the double inequality 1.36 is $0.01578469 \ldots$ 


\section{New Refinements of Young's integral inequality via Pólya's type INTEGRAL INEQUALITIES}

In this section, by virtue of Pólya's type integral inequalities [33, 45, we establish some new refinements in terms of higher order derivatives.

\subsection{Refinements of Young's integral inequality in terms of bounds of the first derivative.}

Theorem 2.1. Let $h(x)$ be a strictly increasing function on $[0, c]$ for $c>0$ and let $h^{-1}$ be the inverse function of $h$. If $h(0)=0, a \in[0, c], b \in[0, h(c)], L$ and $U$ are real constants, and $L \leq h^{\prime}(x) \leq U$ on $(\alpha, \beta)$, then

$$
\begin{aligned}
& \frac{L U\left[a-h^{-1}(b)\right]^{2}-2\left[a-h^{-1}(b)\right][L h(a)-U b]+[h(a)-b]^{2}}{2(U-L)} \\
\leq & \int_{0}^{a} h(x) \mathrm{d} x+\int_{0}^{b} h^{-1}(x) \mathrm{d} x-b h^{-1}(b) \\
\leq & -\frac{L U\left[a-h^{-1}(b)\right]^{2}-2\left[a-h^{-1}(b)\right][U h(a)-L b]+[h(a)-b]^{2}}{2(U-L)} .
\end{aligned}
$$

Proof. Let $f(x)$ be continuous on $[a, b]$ and differentiable on $(a, b)$. If $f(x)$ is not identically a constant and $m \leq f^{\prime}(x) \leq M$ in $(a, b)$, then

$$
\begin{aligned}
& \left|\frac{1}{b-a} \int_{a}^{b} f(x) \mathrm{d} x-\frac{f(a)+f(b)}{2}\right| \\
& \leq \frac{(M-m)(b-a)}{2}\left[\frac{1}{4}-\frac{\left(\frac{f(b)-f(a)}{b-a}-\frac{M+m}{2}\right)^{2}}{(M-m)^{2}}\right] .
\end{aligned}
$$

The inequality 2.2 can be rearranged as a double inequality

$$
\begin{array}{r}
\frac{m M(b-a)^{2}-2(b-a)[m f(b)-M f(a)]+[f(b)-f(a)]^{2}}{2(M-m)} \leq \int_{a}^{b} f(x) \mathrm{d} x \\
\leq-\frac{m M(b-a)^{2}-2(b-a)[M f(b)-m f(a)]+[f(b)-f(a)]^{2}}{2(M-m)} .
\end{array}
$$

These inequalities can be found in [1, Theorem 2], the papers [8, 9, 10, 11, 12, [32, Proposition 2], [33, Section 5] and closely related references therein.

The area $C$ can be computed by 1.7 in Remark 1.2 , which can be estimated, by applying the double inequality $(2.3)$, as

$$
\begin{array}{r}
\frac{L U\left[a-h^{-1}(b)\right]^{2}-2\left[a-h^{-1}(b)\right][L h(a)-U b]+[h(a)-b]^{2}}{2(U-L)} \leq \int_{h^{-1}(b)}^{a} h(x) \mathrm{d} x \\
\leq-\frac{L U\left[a-h^{-1}(b)\right]^{2}-2\left[a-h^{-1}(b)\right][U h(a)-L b]+[h(a)-b]^{2}}{2(U-L)} .
\end{array}
$$

Since

$$
\int_{h^{-1}(b)}^{a} h(x) \mathrm{d} x-b\left[a-h^{-1}(b)\right]=\int_{0}^{a} h(x) \mathrm{d} x+\int_{0}^{b} h^{-1}(x) \mathrm{d} x-a b,
$$


that is,

$$
\int_{h^{-1}(b)}^{a} h(x) \mathrm{d} x=\int_{0}^{a} h(x) \mathrm{d} x+\int_{0}^{b} h^{-1}(x) \mathrm{d} x-b h^{-1}(b),
$$

the double inequality 2.1 follows straightforwardly. The proof of Theorem 2.1 is complete.

\subsection{Refinements of Young's integral inequality in terms of bounds of the second derivative.}

Theorem 2.2. Let $h(x)$ be a strictly increasing function on $[0, c]$ for $c>0$, let $h^{-1}$ be the inverse function of $h$, let $h(0)=0, a \in[0, c]$, and $b \in[0, h(c)]$, and let $L$ and $U$ be real constants such that $L \leq h^{\prime \prime}(x) \leq U$ on $(\alpha, \beta)$. Then

$$
\begin{gathered}
\frac{L\left[a^{3}-\left(h^{-1}(b)\right)^{3}\right]}{6}+\frac{\left(\begin{array}{c}
b-h(a)+a h^{\prime}(a)-h^{-1}(b) h^{\prime}\left(h^{-1}(b)\right) \\
+L\left[\left(h^{-1}(b)\right)^{2}-a^{2}\right] / 2
\end{array}\right)^{2}}{2\left[\left(h^{-1}(b)-a\right) L-h^{\prime}\left(h^{-1}(b)\right)+h^{\prime}(a)\right]} \\
\leq \int_{0}^{a} h(x) \mathrm{d} x+\int_{0}^{b} h^{-1}(x) \mathrm{d} x-a h(a)+\frac{a^{2} h^{\prime}(a)-\left[h^{-1}(b)\right]^{2} h^{\prime}\left(h^{-1}(b)\right)}{2} \\
\leq \frac{U\left[a^{3}-\left(h^{-1}(b)\right)^{3}\right]}{6}+\frac{\left(\begin{array}{c}
b-h(a)+a h^{\prime}(a)-h^{-1}(b) h^{\prime}\left(h^{-1}(b)\right) \\
+U\left[\left(h^{-1}(b)\right)^{2}-a^{2}\right] / 2
\end{array}\right)^{2}}{2\left[\left(h^{-1}(b)-a\right) U-h^{\prime}\left(h^{-1}(b)\right)+h^{\prime}(a)\right]} .
\end{gathered}
$$

Proof. In [29, Corallary] and [41, Corallary 1.2], it was acquired that, if $f(x) \in$ $C([a, b])$ satisfying $N \leq f^{\prime \prime}(x) \leq M$ on $(a, b)$, then

$$
\begin{gathered}
\frac{N\left(b^{3}-a^{3}\right)}{6}+\frac{\left[f(a)-f(b)+b f^{\prime}(b)-a f^{\prime}(a)+N\left(a^{2}-b^{2}\right) / 2\right]^{2}}{2\left[(a-b) N-f^{\prime}(a)+f^{\prime}(b)\right]} \\
\leq \int_{a}^{b} f(x) \mathrm{d} x-b f(b)+a f(a)+\frac{b^{2} f^{\prime}(b)-a^{2} f^{\prime}(a)}{2} \\
\leq \frac{M\left(b^{3}-a^{3}\right)}{6}+\frac{\left[f(a)-f(b)+b f^{\prime}(b)-a f^{\prime}(a)+M\left(a^{2}-b^{2}\right) / 2\right]^{2}}{2\left[(a-b) M-f^{\prime}(a)+f^{\prime}(b)\right]} .
\end{gathered}
$$

Applying the double inequality 2.6 to the integral $\int_{h^{-1}(b)}^{a} h(x) \mathrm{d} x$ and considering Remark 1.2 yield

$$
\begin{aligned}
& \frac{L\left[a^{3}-\left(h^{-1}(b)\right)^{3}\right]}{6}+\frac{\left(\begin{array}{c}
b-h(a)+a h^{\prime}(a)-h^{-1}(b) h^{\prime}\left(h^{-1}(b)\right) \\
+L\left[\left(h^{-1}(b)\right)^{2}-a^{2}\right] / 2
\end{array}\right)^{2}}{2\left[\left(h^{-1}(b)-a\right) L-h^{\prime}\left(h^{-1}(b)\right)+h^{\prime}(a)\right]} \\
& \leq \int_{h^{-1}(b)}^{a} h(x) \mathrm{d} x-a h(a)+b h^{-1}(b)+\frac{a^{2} h^{\prime}(a)-\left[h^{-1}(b)\right]^{2} h^{\prime}\left(h^{-1}(b)\right)}{2} \\
& \leq \frac{U\left[a^{3}-\left(h^{-1}(b)\right)^{3}\right]}{6}+\frac{\left(\begin{array}{c}
b-h(a)+a h^{\prime}(a)-h^{-1}(b) h^{\prime}\left(h^{-1}(b)\right) \\
+U\left[\left(h^{-1}(b)\right)^{2}-a^{2}\right] / 2
\end{array}\right)}{2\left[\left(h^{-1}(b)-a\right) U-h^{\prime}\left(h^{-1}(b)\right)+h^{\prime}(a)\right]} .
\end{aligned}
$$


Substituting (2.4) into (2.7) results in the double inequality (2.5). The proof of Theorem 2.2 is complete.

\subsection{Refinements of Young's integral inequality in terms of bounds of higher order derivatives.}

Theorem 2.3. Let $h(x)$ be a strictly increasing function on $[0, c]$ for $c>0$, let $h^{-1}$ be the inverse of $h$, let $h(0)=0, a \in[0, c]$, and $b \in[0, h(c)]$, and let $h(x)$ have the $(n+1)$-th derivative on $[0, c]$ such that $L \leq h^{(n+1)}(x) \leq U$ on $(\alpha, \beta)$. Then, for all $t$ between $a$ and $h^{-1}(b)$,

(1) when $n$ is a nonnegative odd integer,

$$
\begin{aligned}
& \sum_{i=0}^{n+2} \frac{(-1)^{i}}{i !}\left[S_{n+2}^{(i)}\left(h ; h^{-1}(b), h^{-1}(b), L\right)-S_{n+2}^{(i)}(h ; a, a, L)\right] t^{i} \\
& \leq \int_{0}^{a} h(x) \mathrm{d} x+\int_{0}^{b} h^{-1}(x) \mathrm{d} x-b h^{-1}(b) \\
& \leq \sum_{i=0}^{n+2} \frac{(-1)^{i}}{i !}\left[S_{n+2}^{(i)}\left(h ; h^{-1}(b), h^{-1}(b), U\right)-S_{n+2}^{(i)}(h ; a, a, U)\right] t^{i}
\end{aligned}
$$

(2) when $n$ is a nonnegative even integer,

$$
\begin{gathered}
\sum_{i=0}^{n+2} \frac{(-1)^{i}}{i !}\left[S_{n+2}^{(i)}\left(h ; h^{-1}(b), h^{-1}(b), L\right)-S_{n+2}^{(i)}(h ; a, a, U)\right] t^{i} \\
\leq \int_{0}^{a} h(x) \mathrm{d} x+\int_{0}^{b} h^{-1}(x) \mathrm{d} x-b h^{-1}(b) \\
\leq \sum_{i=0}^{n+2} \frac{(-1)^{i}}{i !}\left[S_{n+2}^{(i)}\left(h ; h^{-1}(b), h^{-1}(b), U\right)-S_{n+2}^{(i)}(h ; a, a, L)\right] t^{i}
\end{gathered}
$$

where $L$ and $U$ are real constants,

$$
S_{n}(h ; u, v, w)=\sum_{k=1}^{n-1} \frac{(-1)^{k}}{k !} u^{k} h^{(k-1)}(v)+(-1)^{n} \frac{w}{n !} u^{n},
$$

and

$$
S_{n}^{(k)}(h ; u, v, w)=\frac{\partial^{k} S_{n}(h ; u, v, w)}{\partial u^{k}} .
$$

Proof. In [29, Theorem], it was discovered that, if $f \in C^{n}([a, b])$ has derivative of $(n+1)$-th order satisfying $N \leq f^{(n+1)}(x) \leq M$ on $(a, b)$, then, for all $t \in(a, b)$,

(1) when $n$ is a nonnegative odd integer,

$$
\begin{aligned}
\sum_{i=0}^{n+2} \frac{(-1)^{i}}{i !}\left[S_{n+2}^{(i)}(f ; a\right. & \left., a, N)-S_{n+2}^{(i)}(f ; b, b, N)\right] t^{i} \leq \int_{a}^{b} f(x) \mathrm{d} x \\
& \leq \sum_{i=0}^{n+2} \frac{(-1)^{i}}{i !}\left[S_{n+2}^{(i)}(f ; a, a, M)-S_{n+2}^{(i)}(f ; b, b, M)\right] t^{i} ;
\end{aligned}
$$

(2) when $n$ is a nonnegative even integer, 


$$
\begin{aligned}
\sum_{i=0}^{n+2} \frac{(-1)^{i}}{i !}\left[S_{n+2}^{(i)}(f ; a\right. & \left., a, N)-S_{n+2}^{(i)}(f ; b, b, M)\right] t^{i} \leq \int_{a}^{b} f(x) \mathrm{d} x \\
& \leq \sum_{i=0}^{n+2} \frac{(-1)^{i}}{i !}\left[S_{n+2}^{(i)}(f ; a, a, M)-S_{n+2}^{(i)}(f ; b, b, N)\right] t^{i} .
\end{aligned}
$$

These inequalities can also be found in [30, 31, 34, 40, 45] and closely related references therein.

Applying 2.10 and 2.11 to the integral $\int_{h^{-1}(b)}^{a} h(x) \mathrm{d} x$ and considering Remark 1.2 yield

(1) when $n$ is a nonnegative odd integer,

$$
\begin{gathered}
\sum_{i=0}^{n+2} \frac{(-1)^{i}}{i !}\left[S_{n+2}^{(i)}\left(h ; h^{-1}(b), h^{-1}(b), L\right)-S_{n+2}^{(i)}(h ; a, a, L)\right] t^{i} \leq \int_{h^{-1}(b)}^{a} h(x) \mathrm{d} x \\
\leq \sum_{i=0}^{n+2} \frac{(-1)^{i}}{i !}\left[S_{n+2}^{(i)}\left(h ; h^{-1}(b), h^{-1}(b), U\right)-S_{n+2}^{(i)}(h ; a, a, U)\right] t^{i} ;
\end{gathered}
$$

(2) when $n$ is a nonnegative even integer,

$$
\begin{gathered}
\sum_{i=0}^{n+2} \frac{(-1)^{i}}{i !}\left[S_{n+2}^{(i)}\left(h ; h^{-1}(b), h^{-1}(b), L\right)-S_{n+2}^{(i)}(h ; a, a, U)\right] t^{i} \leq \int_{h^{-1}(b)}^{a} h(x) \mathrm{d} x \\
\leq \sum_{i=0}^{n+2} \frac{(-1)^{i}}{i !}\left[S_{n+2}^{(i)}\left(h ; h^{-1}(b), h^{-1}(b), U\right)-S_{n+2}^{(i)}(h ; a, a, L)\right] t^{i} .
\end{gathered}
$$

Substituting 2.4 into 2.12 and 2.13 results in 2.8 and 2.9. The proof of Theorem 2.3 is complete.

\subsection{Refinements of Young's integral inequality in terms of $L^{p}$-norms.}

Theorem 2.4. Let $h(x)$ be a strictly increasing function on $[0, c]$ for $c>0$, let $h^{-1}$ be the inverse of $h$, let $h(0)=0, a \in[0, c]$, and $b \in[0, h(c)]$, and let $h(x)$ have the $(n+1)$-th derivative on $[\alpha, \beta]$ such that $h^{(n+1)} \in L^{p}([\alpha, \beta])$ for $p, q>0$ with $\frac{1}{p}+\frac{1}{q}=1$. Then, for all $t \in[\alpha, \beta]$,

(1) when $p, q>1$, we have

$$
\begin{gathered}
\left|\int_{h^{-1}(b)}^{a} h(x) \mathrm{d} x-\sum_{i=0}^{n} \frac{h^{(i)}\left(h^{-1}(b)\right)}{(i+1) !}\left(t-h^{-1}(b)\right)^{i+1}+\sum_{i=0}^{n} \frac{h^{(i)}(a)}{(i+1) !}(t-a)^{i+1}\right| \\
\leq \frac{\left(t-h^{-1}(b)\right)^{n+1+1 / q}+(a-t)^{n+1+1 / q}}{(n+1) ! \sqrt[q]{n q+q+1}}\left\|h^{(n+1)}\right\|_{L^{p}\left(\left[h^{-1}(b), a\right]\right)} \\
\leq \frac{2\left(a-h^{-1}(b)\right)^{n+2}}{(n+1) !}\left\|h^{(n+1)}\right\|_{L^{p}\left(\left[h^{-1}(b), a\right]\right)}
\end{gathered}
$$

(2) when $p=\infty$, we have

$$
\left|\int_{h^{-1}(b)}^{a} h(x) \mathrm{d} x-\sum_{i=0}^{n} \frac{h^{(i)}\left(h^{-1}(b)\right)}{(i+1) !}\left(t-h^{-1}(b)\right)^{i+1}+\sum_{i=0}^{n} \frac{h^{(i)}(a)}{(i+1) !}(t-a)^{i+1}\right|
$$




$$
\begin{aligned}
& \leq \frac{\left(t-h^{-1}(b)\right)^{n+2}+(a-t)^{n+2}}{(n+2) !}\left\|h^{(n+1)}\right\|_{L^{\infty}\left(\left[h^{-1}(b), a\right]\right)} \\
& \leq \frac{2\left(a-h^{-1}(b)\right)^{n+2}}{(n+2) !}\left\|h^{(n+1)}\right\|_{L^{\infty}\left(\left[h^{-1}(b), a\right]\right)} ;
\end{aligned}
$$

(3) when $p=1$, we have

$$
\begin{aligned}
\mid \int_{h^{-1}(b)}^{a} h(x) \mathrm{d} x-\sum_{i=0}^{n} & \frac{h^{(i)}\left(h^{-1}(b)\right)}{(i+1) !}\left(t-h^{-1}(b)\right)^{i+1}+\sum_{i=0}^{n} \frac{h^{(i)}(a)}{(i+1) !}(t-a)^{i+1} \mid \\
& \leq \frac{\left(t-h^{-1}(b)\right)^{n+1}+(a-t)^{n+1}}{(n+1) !}\left\|h^{(n+1)}\right\|_{L\left(\left[h^{-1}(b), a\right]\right)} \\
& \leq \frac{2\left(a-h^{-1}(b)\right)^{n+1}}{(n+1) !}\left\|h^{(n+1)}\right\|_{L\left(\left[h^{-1}(b), a\right]\right)} .
\end{aligned}
$$

Proof. Let $f \in C^{n}([a, b])$ have derivative of $(n+1)$-th order on $(a, b)$ and $f^{(n+1)} \in$ $L^{p}([a, b])$ for positive numbers $p$ and $q$ satisfying $\frac{1}{p}+\frac{1}{q}=1$. In [16] and [17, Theorem 2], it was established that, for any $t \in(a, b)$,

(1) when $p, q>1$, we have

$$
\begin{aligned}
\left|\int_{a}^{b} f(x) \mathrm{d} x-\sum_{i=0}^{n} \frac{f^{(i)}(a)}{(i+1) !}(t-a)^{i+1}+\sum_{i=0}^{n} \frac{f^{(i)}(b)}{(i+1) !}(t-b)^{i+1}\right| \\
\leq \frac{(t-a)^{n+1+1 / q}+(b-t)^{n+1+1 / q}}{(n+1) ! \sqrt[q]{n q+q+1}}\left\|f^{(n+1)}\right\|_{L^{p}([a, b])} \\
\leq \frac{2(b-a)^{n+2}}{(n+1) !}\left\|f^{(n+1)}\right\|_{L^{p}([a, b])} ;
\end{aligned}
$$

(2) when $p=\infty$, we have

$$
\begin{aligned}
\mid \int_{a}^{b} f(x) \mathrm{d} x-\sum_{i=0}^{n} \frac{f^{(i)}(a)}{(i+1) !}(t & -a)^{i+1}+\sum_{i=0}^{n} \frac{f^{(i)}(b)}{(i+1) !}(t-b)^{i+1} \mid \\
& \leq \frac{(t-a)^{n+2}+(b-t)^{n+2}}{(n+2) !}\left\|f^{(n+1)}\right\|_{L^{\infty}([a, b])} \\
& \leq \frac{2(b-a)^{n+2}}{(n+2) !}\left\|f^{(n+1)}\right\|_{L^{\infty}([a, b])} ;
\end{aligned}
$$

(3) when $p=1$, we have

$$
\begin{aligned}
\left|\int_{a}^{b} f(x) \mathrm{d} x-\sum_{i=0}^{n} \frac{f^{(i)}(a)}{(i+1) !}(t-a)^{i+1}+\sum_{i=0}^{n} \frac{f^{(i)}(b)}{(i+1) !}(t-b)^{i+1}\right| \\
\leq \frac{(t-a)^{n+1}+(b-t)^{n+1}}{(n+1) !}\left\|f^{(n+1)}\right\|_{L([a, b])} \\
\leq \frac{2(b-a)^{n+1}}{(n+1) !}\left\|f^{(n+1)}\right\|_{L([a, b])} .
\end{aligned}
$$

Applying three inequalities 2.17), 2.18, and 2.19) to the integral $\int_{h^{-1}(b)}^{a} h(x) \mathrm{d} x$ and considering Remark 1.2 lead to the following conclusions: 
(1) when $p, q>1$, we have

$$
\begin{gathered}
\left|\int_{h^{-1}(b)}^{a} h(x) \mathrm{d} x-\sum_{i=0}^{n} \frac{h^{(i)}\left(h^{-1}(b)\right)}{(i+1) !}\left(t-h^{-1}(b)\right)^{i+1}+\sum_{i=0}^{n} \frac{h^{(i)}(a)}{(i+1) !}(t-a)^{i+1}\right| \\
\leq \frac{\left(t-h^{-1}(b)\right)^{n+1+1 / q}+(a-t)^{n+1+1 / q}}{(n+1) ! \sqrt[q]{n q+q+1}}\left\|h^{(n+1)}\right\|_{L^{p}\left(\left[h^{-1}(b), a\right]\right)} \\
\leq \frac{2\left(a-h^{-1}(b)\right)^{n+2}}{(n+1) !}\left\|h^{(n+1)}\right\|_{L^{p}\left(\left[h^{-1}(b), a\right]\right)}
\end{gathered}
$$

(2) when $p=\infty$, we have

$$
\begin{aligned}
\mid \int_{h^{-1}(b)}^{a} h(x) \mathrm{d} x- & \sum_{i=0}^{n} \frac{h^{(i)}\left(h^{-1}(b)\right)}{(i+1) !}\left(t-h^{-1}(b)\right)^{i+1}+\sum_{i=0}^{n} \frac{h^{(i)}(a)}{(i+1) !}(t-a)^{i+1} \mid \\
& \leq \frac{\left(t-h^{-1}(b)\right)^{n+2}+(a-t)^{n+2}}{(n+2) !}\left\|h^{(n+1)}\right\|_{L^{\infty}\left(\left[h^{-1}(b), a\right]\right)} \\
& \leq \frac{2\left(a-h^{-1}(b)\right)^{n+2}}{(n+2) !}\left\|h^{(n+1)}\right\|_{L^{\infty}\left(\left[h^{-1}(b), a\right]\right)}
\end{aligned}
$$

(3) when $p=1$, we have

$$
\begin{gathered}
\left|\int_{h^{-1}(b)}^{a} h(x) \mathrm{d} x-\sum_{i=0}^{n} \frac{h^{(i)}\left(h^{-1}(b)\right)}{(i+1) !}\left(t-h^{-1}(b)\right)^{i+1}+\sum_{i=0}^{n} \frac{h^{(i)}(a)}{(i+1) !}(t-a)^{i+1}\right| \\
\leq \frac{\left(t-h^{-1}(b)\right)^{n+1}+(a-t)^{n+1}}{(n+1) !}\left\|h^{(n+1)}\right\|_{L\left(\left[h^{-1}(b), a\right]\right)} \\
\leq \frac{2\left(a-h^{-1}(b)\right)^{n+1}}{(n+1) !}\left\|h^{(n+1)}\right\|_{L\left(\left[h^{-1}(b), a\right]\right)} .
\end{gathered}
$$

Substituting (2.4) into 2.20, 2.21, and 2.22 results in (2.14), 2.15), and (2.16). The proof of Theorem 2.4 is complete.

\subsection{Three examples for new refinements of Young's integral inequalities.}

2.5.1. First example. Let $h(x)=\sqrt[4]{x^{4}+1}-1$ and let $a=3$ and $b=2$ in Theorem 2.1. Then

$$
\begin{gathered}
h^{\prime}(x)=\frac{x^{3}}{\left(x^{4}+1\right)^{3 / 4}}, \quad h^{\prime \prime}(x)=\frac{3 x^{2}}{\left(x^{4}+1\right)^{7 / 4}}>0, \\
h^{-1}(2)=\left(3^{4}-1\right)^{1 / 4}=2 \sqrt[4]{5}=2.990 \ldots, \\
L=h^{\prime}(2 \sqrt[4]{5})=\frac{8 \times 5^{3 / 4}}{27}, \quad U=h^{\prime}(3)=\frac{27}{82^{3 / 4}}, \\
h(3)=\sqrt[4]{82}-1, \quad \int_{0}^{3} h(x) \mathrm{d} x=\int_{0}^{3} \sqrt[4]{x^{4}+1} \mathrm{~d} x-3, \\
\int_{0}^{2} h^{-1}(x) \mathrm{d} x=\int_{0}^{2} \sqrt[4]{(x+1)^{4}-1}=\int_{1}^{3} \sqrt[4]{x^{4}-1} \mathrm{~d} x,
\end{gathered}
$$


and

$$
\begin{gathered}
\frac{\left(\begin{array}{c}
\frac{8 \times 5^{3 / 4}}{27} \frac{27}{82^{3 / 4}}(3-2 \sqrt[4]{5})^{2}+(\sqrt[4]{82}-3)^{2} \\
-2(3-2 \sqrt[4]{5})\left[\frac{8 \times 5^{3 / 4}}{27}(\sqrt[4]{82}-1)-\frac{2 \times 27}{82^{3 / 4}}\right]
\end{array}\right)}{2\left(\frac{27}{82^{3 / 4}}-\frac{8 \times 5^{3 / 4}}{27}\right)} \\
\leq \int_{0}^{3} \sqrt[4]{x^{4}+1} \mathrm{~d} x+\int_{1}^{3} \sqrt[4]{x^{4}-1} \mathrm{~d} x-3-4 \sqrt[4]{5} \\
\leq-\frac{\left(\frac{8 \times 5^{3 / 4}}{27} \frac{27}{82^{3 / 4}}(3-2 \sqrt[4]{5})^{2}+(\sqrt[4]{82}-3)^{2}\right.}{-2(3-2 \sqrt[4]{5})\left[\frac{27}{82^{3 / 4}}(\sqrt[4]{82}-1)-\frac{2 \times 8 \times 5^{3 / 4}}{27}\right]} \\
2\left(\frac{27}{82^{3 / 4}}-\frac{8 \times 5^{3 / 4}}{27}\right)
\end{gathered}
$$

Consequently, we arrive at

$$
\begin{aligned}
& 9.00004286765564673 \ldots<\int_{0}^{3} \sqrt[4]{x^{4}+1} \mathrm{~d} x+\int_{1}^{3} \sqrt[4]{x^{4}-1} \mathrm{~d} x \\
&<9.00004287010602764 \ldots
\end{aligned}
$$

which is neither the best nor the weakest estimate among those in Section 1.11 . The gap between the upper and lower bounds in the double inequality (2.23) is $0.0000000024506 \ldots$ which, comparing with those gaps in Section 1.11, is neither the smallest nor the biggest one.

\subsubsection{Second example. Let}

$$
h(x)= \begin{cases}e^{-1 / x}, & x>0 \\ 0, & x=0 .\end{cases}
$$

Let $a=b=\frac{1}{2}$ in Theorem 2.1. Then

$$
\begin{gathered}
h^{\prime}(x)=\frac{e^{-1 / x}}{x^{2}}, \quad h^{\prime \prime}(x)=\frac{e^{-1 / x}(1-2 x)}{x^{4}}, \\
h^{-1}\left(\frac{1}{2}\right)=\frac{1}{\ln 2}=1.44 \ldots, \quad h\left(\frac{1}{2}\right)=\frac{1}{e^{2}}, \\
U=h^{\prime}\left(\frac{1}{2}\right)=\frac{4}{e^{2}}=0.54134 \ldots, \quad L=h^{\prime}\left(\frac{1}{\ln 2}\right)=\frac{\ln ^{2} 2}{2}=0.24022 \ldots,
\end{gathered}
$$

and

$$
\begin{gathered}
\frac{\frac{\ln ^{2} 2}{2} \frac{4}{e^{2}}\left(\frac{1}{2}-\frac{1}{\ln 2}\right)^{2}-2\left(\frac{1}{2}-\frac{1}{\ln 2}\right)\left(\frac{\ln ^{2} 2}{2} \frac{1}{e^{2}}-\frac{4}{e^{2}} \frac{1}{2}\right)+\left(\frac{1}{e^{2}}-\frac{1}{2}\right)^{2}}{2\left(\frac{4}{e^{2}}-\frac{\ln ^{2} 2}{2}\right)} \\
\leq \int_{0}^{1 / 2} \frac{1}{e^{1 / x}} \mathrm{~d} x-\int_{0}^{1 / 2} \frac{1}{\ln x} \mathrm{~d} x-\frac{1}{2 \ln 2} \\
\leq-\frac{\frac{\ln ^{2} 2}{2} \frac{4}{e^{2}}\left(\frac{1}{2}-\frac{1}{\ln 2}\right)^{2}-2\left(\frac{1}{2}-\frac{1}{\ln 2}\right)\left(\frac{4}{e^{2}} \frac{1}{e^{2}}-\frac{\ln ^{2} 2}{2} \frac{1}{2}\right)+\left(\frac{1}{e^{2}}-\frac{1}{2}\right)^{2}}{2\left(\frac{4}{e^{2}}-\frac{\ln ^{2} 2}{2}\right)} .
\end{gathered}
$$

Accordingly, it follows that 


$$
\begin{aligned}
0.388457763460961578 \ldots<\int_{0}^{1 / 2} \frac{1}{e^{1 / x}} \mathrm{~d} x & -\int_{0}^{1 / 2} \frac{1}{\ln x} \mathrm{~d} x \\
& <0.455309856619062079 \ldots
\end{aligned}
$$

whose lower bound is better, but whose upper bound is worse, than the corresponding ones in 1.35 . The gap between the upper and lower bounds in the double inequality (2.24) is $0.066852093209446 \ldots$ which is bigger than the gap $0.057414764502015 \ldots$ in the double inequality 1.35 .

2.5.3. Third example. Let $h(x)=e^{x^{2}}-1$ for $x \geq 0$. Then $h^{-1}(x)=\sqrt{\ln (1+x)}$ for $x \geq 0$. Let $a=b=1$ in Theorem 2.1. Then

$$
\begin{gathered}
h^{\prime}(x)=2 x e^{x^{2}}, \quad h^{-1}(1)=\sqrt{\ln 2}=0.83255 \ldots, \quad h(1)=e-1, \\
U=h^{\prime}(1)=2 e=5.4365 \ldots, \quad L=h^{\prime}(\sqrt{\ln 2})=4 \sqrt{\ln 2}=3.3302 \ldots,
\end{gathered}
$$

and

$$
\begin{gathered}
\frac{8 e \sqrt{\ln 2}(1-\sqrt{\ln 2})^{2}-2(1-\sqrt{\ln 2})[4 \sqrt{\ln 2}(e-1)-2 e]+(e-2)^{2}}{2(2 e-4 \sqrt{\ln 2})} \\
\leq \int_{0}^{1}\left(e^{x^{2}}-1\right) \mathrm{d} x+\int_{0}^{1} \sqrt{\ln (1+x)} \mathrm{d} x-\sqrt{\ln 2} \\
\leq-\frac{8 e \sqrt{\ln 2}(1-\sqrt{\ln 2})^{2}-2(1-\sqrt{\ln 2})[2 e(e-1)-4 \sqrt{\ln 2}]+(e-2)^{2}}{2(2 e-4 \sqrt{\ln 2})} .
\end{gathered}
$$

As a result, we have

$$
\begin{aligned}
2.05281277502489567 \ldots \leq \int_{0}^{1} e^{x^{2}} \mathrm{~d} x+\int_{0}^{1} & \sqrt{\ln (1+x)} \mathrm{d} x \\
& \leq 2.06746020503978898 \ldots
\end{aligned}
$$

whose lower bound is better, but whose upper bound is worse, than the corresponding ones in (1.36). The gap between the upper and lower bounds in the double inequality (2.25) is $0.01464743001489 \ldots$ which is smaller than the corresponding gap $0.01578469 \ldots$ in the double inequality 1.36 .

\section{More REMARKS}

Finally, we would like to list more remarks on our main results and possible developing directions.

Remark 3.1. Theorems 2.1 and 2.2 are special cases of Theorem2.3 In other words, Theorems 2.1 and 2.2 can be deduced from Theorem 2.3 .

Remark 3.2. Some Taylor-like power expansions such as those in [15, 22, 36, 39. and closely related references can be used to refine Young's integral inequality (1.1).

Remark 3.3. At the present position, we conclude that many estimates of definite integrals can be used to refine Young's integral inequality (1.1).

Remark 3.4. Essentially speaking, all refinements in this paper are estimates of the area $C$ which can be geometrically demonstrated in Figures 1 to 6 and analytically expressed by 1.7 in Remark 1.2 . 


\section{REFERENCES}

[1] R. P. Agarwal and S. S. Dragomir, An application of Hayashi inequality for differentiable functions, Computers Math. Appl. 32 (1996), no. 6, 95-99; available online at https://doi. org/10.1016/0898-1221(96)00146-0.

[2] H. Alzer and M. K. Kwong, On Young's inequality, J. Math. Anal. Appl. 469 (2019), no. 2, 480-492; available online at https://doi.org/10.1016/j.jmaa.2018.06.061.

[3] H. Alzer and S. Koumandos, A new refinement of Young's inequality, Proc. Edinb. Math. Soc. (2) 50 (2007), no. 2, 255-262; available online at https://doi.org/10.1017/ S0013091504000744

[4] D. R. Anderson, Young's integral inequality on time scales revisited, J. Inequal. Pure Appl. Math. 8 (2007), no. 3, Art. 64; available online at http://www.emis.de/journals/JIPAM/ article876.html

[5] T. M. Apostol, Calculus, Vol. I: One-variable calculus, with an introduction to linear algebra; Second edition, Blaisdell Publishing Co. Ginn and Co., Waltham, Mass.-Toronto, Ont.-London, 1967.

[6] T. M. Apostol, Mathematical Analysis, Second edition, Addison-Wesley Publishing Co., Reading, Mass.-London-Don Mills, Ont., 1974.

[7] S.-P. Bai, S.-H. Wang, and F. Qi, On HT-convexity and Hadamard-type inequalities, J. Inequal. Appl. 2020, Paper No. 3, 12 pages; available online at https://doi.org/10.1186/ s13660-019-2276-3

[8] P. Cerone, Generalised trapezoidal rules with error involving bounds of the $n$-th derivative, Math. Inequal. Appl. 5 (2002), no. 3, 451-462; available online at https://doi.org/10.7153/ mia-05-44

[9] P. Cerone, On Gini mean difference bounds via generalised Iyengar results, Hacet. J. Math. Stat. 44 (2015), no. 4, 789-799; available online at https://doi.org/10.15672/HJMS. 2015449430 .

[10] P. Cerone and S. S. Dragomir, Lobatto type quadrature rules for functions with bounded derivative, Math. Inequal. Appl. 3 (2000), no. 2, 197-209; available online at https://doi. org/10.7153/mia-03-23

[11] P. Cerone and S. S. Dragomir, On a weighted generalization of Iyengar type inequalities involving bounded first derivative, Math. Inequal. Appl. 3 (2000), no. 1, 35-44; available online at https://doi.org/10.7153/mia-03-04

[12] X.-L. Cheng, The Iyengar-type inequality, Appl. Math. Lett. 14 (2001), no. 8, 975-978; available online at https://doi.org/10.1016/S0893-9659(01)00074-X

[13] S. S. Dragomir, A note on Young's inequality, Rev. R. Acad. Cienc. Exactas Fís. Nat. Ser. A Mat. RACSAM 111 (2017), no. 2, 349-354; available online at https://doi.org/10.1007/ s13398-016-0300-8

[14] S. S. Dragomir, Trace inequalities for positive operators via recent refinements and reverses of Young's inequality, Spec. Matrices 6 (2018), 180-192; available online at https://doi. org/10.1515/spma-2018-0015

[15] S. S. Dragomir, F. Qi, G. Hanna, and P. Cerone, New Taylor-like expansions for functions of two variables and estimates of their remainders, J. Korean Soc. Indust. Appl. Math. 9 (2005), no. 2, 1-16.

[16] B.-N. Guo and F. Qi, Estimates for an integral in $L^{p}$ norm of the $(n+1)$-th derivative of its integrand, Inequality Theory and Applications, Volume 3, 127-131, Nova Science Publishers, Hauppauge, NY, 2003.

[17] B.-N. Guo and F. Qi, Some estimates of an integral in terms of the $L^{p}$-norm of the $(n+1)$ st derivative of its integrand, Anal. Math. 29 (2003), no. 1, 1-6; available online at http: //dx.doi.org/10.1023/A:1022894413541

[18] A. Hoorfar and F. Qi, A new refinement of Young's inequality, Math. Inequal. Appl. 11 (2008), no. 4, 689-692; available online at https://doi.org/10.7153/mia-11-58

[19] J. Jakšetić and J. Pečarić, An estimation of Young inequality, Asian-Eur. J. Math. 2 (2009), no. 4, 593-604; available online at https://doi.org/10.1142/S1793557109000509.

[20] J. Jakšetić and J. Pečarić, A note on Young inequality, Math. Inequal. Appl. 13 (2010), no. 1, 43-48; available online at https://doi.org/10.7153/mia-13-03

[21] P. Kórus, A refinement of Young's inequality, Acta Math. Hungar. 153 (2017), no. 2, 430-435; available online at https://doi.org/10.1007/s10474-017-0735-1 
[22] Q.-M. Luo, F. Qi, and B.-N. Guo, K. Petr's formula of double integral and estimates of its remainder, Int. J. Math. Sci. 3 (2004), no. 1, 77-92.

[23] P. R. Mercer, Error terms for Steffensen's, Young's and Chebychev's inequalities, J. Math. Inequal. 2 (2008), no. 4, 479-486; available online at https://doi.org/10.7153/jmi-02-43

[24] P. R. Mercer, Techniques of Integration, Chapter 11 in: More Calculus of a Single Variable, Undergraduate Texts in Mathematics, Springer, New York, 2014; available online at https: //doi.org/10.1007/978-1-4939-1926-0_11

[25] D. S. Mitrinović, Analytic Inequalities, In cooperation with P. M. Vasić, Die Grundlehren der mathematischen Wissenschaften, Band 165, Springer-Verlag, New York-Berlin, 1970.

[26] D. S. Mitrinović, J. E. Pečarić, and A. M. Fink, Classical and New Inequalities in Analysis, Kluwer Academic Publishers, 1993; available online at http://dx.doi.org/10.1007/ 978-94-017-1043-5

[27] F. W. J. Olver, D. W. Lozier, R. F. Boisvert, and C. W. Clark (eds.), NIST Handbook of Mathematical Functions, Cambridge University Press, New York, 2010; available online at http://dlmf.nist.gov/

[28] B. G. Pachpatte, On some inequalities for convex functions, RGMIA Res. Rep. Coll. 6 (2003), Suppl., Art. 1, 9 pages; available online at http://rgmia.org/v6(E).php.

[29] F. Qi, Further generalizations of inequalities for an integral, Univ. Beograd. Publ. Elektrotehn. Fak. Ser. Mat. 8 (1997), 79-83.

[30] F. Qi, Inequalities for a multiple integral, Acta Math. Hungar. 84 (1999), no. 1-2, 19-26; available online at https://doi.org/10.1023/A:1006642601341

[31] F. Qi, Inequalities for a weighted multiple integral, J. Math. Anal. Appl. 253 (2001), no. 2, 381-388; available online at https://doi.org/10.1006/jmaa.2000.7138

[32] F. Qi, Inequalities for an integral, Math. Gaz. 80 (1996), no. 488, 376-377; available online at https://doi.org/10.2307/3619581

[33] F. Qi, Pólya type integral inequalities: origin, variants, proofs, refinements, generalizations, equivalences, and applications, Math. Inequal. Appl. 18 (2015), no. 1, 1-38; available online at http://dx.doi.org/10.7153/mia-18-01

[34] F. Qi, P. Cerone, and S. S. Dragomir, Some new Iyengar type inequalities, Rocky Mountain J. Math. 35 (2005), no. 3, 997-1015; available online at https://doi.org/10.1216/rmjm/ 1181069718 .

[35] F. Qi, L.-H. Cui, and S.-L. Xu, Some inequalities constructed by Tchebysheff's integral inequality, Math. Inequal. Appl. 2 (1999), no. 4, 517-528; available online at http: //dx.doi.org/10.7153/mia-02-42

[36] F. Qi, Q.-M. Luo, and B.-N. Guo, Darboux's formula with integral remainder of functions with two independent variables, Appl. Math. Comput. 199 (2008), no. 2, 691-703; available online at https://doi.org/10.1016/j.amc.2007.10.028

[37] F. Qi, P. O. Mohammed, J.-C. Yao, and Y.-H. Yao, Generalized fractional integral inequalities of Hermite-Hadamard type for $(\alpha, m)$-convex functions, J. Inequal. Appl. 2019, Paper No. 135, 17 pages; available online at https://doi.org/10.1186/s13660-019-2079-6

[38] F. Qi, G. Rahman, S. M. Hussain, W.-S. Du, and Kottakkaran Sooppy Nisar, Some inequalities of Čebyšev type for conformable $k$-fractional integral operators, Symmetry 10 (2018), no. 11, Article 614, 8 pages; available online at https://doi.org/10.3390/sym10110614

[39] F. Qi and W. Ul-Haq, Some integral inequalities involving the expectation and variance via Darboux's expansion, Adv. Appl. Math. Sci. 18 (2019), no. 7, 545-552.

[40] F. Qi, Z.-L. Wei, and Q. Yang, Generalizations and refinements of Hermite-Hadamard's inequality, Rocky Mountain J. Math. 35 (2005), no. 1, 235-251; available online at http: //dx.doi.org/10.1216/rmjm/1181069779

[41] F. Qi and Y.-J. Zhang, Inequalities for a weighted integral, Adv. Stud. Contemp. Math. (Kyungshang) 4 (2002), no. 2, 93-101.

[42] D. Ruthing, On Young's inequality, Internat. J. Math. Ed. Sci. Techn. 25 (1994), no. 2, 161-164; available online at https://doi.org/10.1080/0020739940250201

[43] J. Sándor and V. E. S. Szabó, On an inequality for the sum of infimums of functions, J. Math. Anal. Appl. 204 (1996), no. 3, 646-654; available online at https://doi.org/10.1006/jmaa. 1996.0459

[44] Y. Shuang and F. Qi, Integral inequalities of Hermite-Hadamard type for extended s-convex functions and applications, Mathematics 6 (2018), no. 11, Article 223, 12 pages; available online at https://doi.org/10.3390/math6110223 
[45] Y. Sun, H.-T. Yang, and F. Qi, Some inequalities for multiple integrals on the $n$-dimensional ellipsoid, spherical shell, and ball, Abstr. Appl. Anal. 2013 (2013), Article ID 904721, 8 pages; available online at https://doi.org/10.1155/2013/904721

[46] T. Takahashi, Remarks on some inequalities, Tôhoku Math. J. 36 (1932), 99-106.

[47] J.-F. Tian, Extension of Hu Ke's inequality and its applications, J. Inequal. Appl. 2011, 2011:77, 14 pages; available online at https://doi.org/10.1186/1029-242X-2011-77

[48] J.-F. Tian and M.-H. Ha, Properties of generalized sharp Hölder's inequalities, J. Math. Inequal. 11 (2017), no. 2, 511-525; available online at https://doi.org/10.7153/jmi-11-42

[49] J.-Q. Wang, B.-N. Guo, and F. Qi, Generalizations and applications of Young's integral inequality by higher order derivatives, J. Inequal. Appl. 2019, Paper No. 243, 18 pages; available online at https://doi.org/10.1186/s13660-019-2196-2

[50] A. Witkowski, On Young inequality, J. Inequal. Pure Appl. Math. 7 (2006), no. 5, Art. 164; available online at http://www.emis.de/journals/JIPAM/article782.html

[51] Y. Wu, F. Qi, and D.-W. Niu, Integral inequalities of Hermite-Hadamard type for the product of strongly logarithmically convex and other convex functions, Maejo Int. J. Sci. Technol. 9 (2015), no. 3, 394-402.

[52] H.-P. Yin and F. Qi, Hermite-Hadamard type inequalities for the product of $(\alpha, m)$-convex functions, J. Nonlinear Sci. Appl. 8 (2015), no. 3, 231-236; available online at https://doi. org/10.22436/jnsa.008.03.07.

[53] H.-P. Yin and F. Qi, Hermite-Hadamard type inequalities for the product of $(\alpha, m)$ convex functions, Missouri J. Math. Sci. 27 (2015), no. 1, 71-79; available online at http://projecteuclid.org/euclid.mjms/1449161369

[54] W. H. Young, On classes of summable functions and their Fourier series, Proc. Roy. Soc. London Ser. A 87 (1912), 225-229; available online at https://doi.org/10.1098/rspa.1912. 0076

[55] L. Zhu, On Young's inequality, Internat. J. Math. Ed. Sci. Tech. 35 (2004), no. 4, 601-603; available online at https://doi.org/10.1080/00207390410001686698

(Qi) College of Mathematics, Inner Mongolia University for Nationalities, Tongliao 028043, Inner Mongolia, China; Institute of Mathematics, Henan Polytechnic UniverSity, Jiaozuo 454010, Henan, China; School of Mathematical Sciences, Tianjin PolytechNiC University, Tianjin 300387, China

E-mail address: qifeng618@gmail.com, qifeng618@hotmail.com, qifeng618@qq.com

$U R L:$ https://qifeng618. wordpress.com

(Li) Department of Fundamental Courses, Zhenghzou University of Science and TechNOLOGy, Zhengzhou 450064, Henan, China

E-mail address: wen.hui.li@foxmail.com, wen.hui.li102@gmail.com

$U R L:$ https://orcid.org/0000-0002-1848-8855

(Wu) School of Computer Science, Sichuan Technology and Business University, Chengdu 611745, Sichuan, China

E-mail address: mrwuguosheng@sina.com

(Guo) School of Mathematics and Informatics, Henan Polytechnic University, Jiaozuo 454010, Henan, China

E-mail address: bai.ni.guo@gmail.com, bai.ni.guo@hotmail.com

$U R L$ : https://orcid.org/0000-0001-6156-2590 